\title{
Acute kidney injury
}

John A. Kellum (1) ', Paola Romagnani², Gloria Ashuntantang ${ }^{3}$, Claudio Ronco $\mathbb{1}^{4,5}$, Alexander Zarbock ${ }^{6}$ and Hans-Joachim Anders (10 ${ }^{7 凶}$

Abstract $\mid$ Acute kidney injury (AKI) is defined by a sudden loss of excretory kidney function. AKI is part of a range of conditions summarized as acute kidney diseases and disorders (AKD), in which slow deterioration of kidney function or persistent kidney dysfunction is associated with an irreversible loss of kidney cells and nephrons, which can lead to chronic kidney disease (CKD). New biomarkers to identify injury before function loss await clinical implementation. AKI and AKD are a global concern. In low-income and middle-income countries, infections and hypovolaemic shock are the predominant causes of AKI. In high-income countries, AKI mostly occurs in elderly patients who are in hospital, and is related to sepsis, drugs or invasive procedures. Infection and trauma-related AKI and AKD are frequent in all regions. The large spectrum of AKI implies diverse pathophysiological mechanisms. AKI management in critical care settings is challenging, including appropriate volume control, nephrotoxic drug management, and the timing and type of kidney support. Fluid and electrolyte management are essential. As $\mathrm{AKI}$ can be lethal, kidney replacement therapy is frequently required. AKI has a poor prognosis in critically ill patients. Long-term consequences of AKI and AKD include CKD and cardiovascular morbidity. Thus, prevention and early detection of AKI are essential.

Acute kidney injury (AKI) describes a sudden loss of kidney function that is determined on the basis of increased serum creatinine levels (a marker of kidney excretory function) and reduced urinary output (oliguria) (a quantitative marker of urine production) and is limited to a duration of 7 days (TABLE 1) $)^{1}$. AKI is part of a variety of functional kidney conditions, which are summarized as acute kidney disease and disorders (AKD) and can range from mild and self-limiting to severe and persistent. AKD can occur without ever meeting the criterion of rapid onset of AKI, for example when kidney dysfunction evolves slowly ${ }^{1}$, or AKD can continue after an AKI event has ended, for example, when kidney dysfunction does not resolve or when structural damage to the kidney persists. By definition, AKD persisting for $>3$ months is referred to as chronic kidney disease (CKD). Of note, AKI and AKD frequently occur in patients with precedent CKD (FIG. 1).

As the diagnostic markers serum creatinine and urine output level measure loss of kidney function and not injury, AKI can be seen as a misnomer. In the absence of injury markers, individuals with episodes of transient volume depletion can meet the diagnostic criteria of AKI without injury being present. A few hours of volume depletion in an otherwise healthy human may have no long-term health implications. Similarly, reninangiotensin system inhibitors or other drugs that affect glomerular filtration may result in small changes in serum creatinine levels that are not indicative of kidney injury ${ }^{2}$. However, AKI persisting despite volume therapy probably indicates structural damage to the kidney ${ }^{3}$.

Unfortunately, direct assessment of kidney damage, apart from biopsy, is not possible with existing technology; hence, numerous urinary biomarkers are in use or have been proposed as indicators of glomerular or tubular cell injury ${ }^{4}$. A consensus statement published in 2020 suggested that damage biomarkers should be integrated into the definition of AKI to augment its classification (TABLE 2) $)^{5}$ Importantly, both functional impairment (serum creatinine level elevation and/or urine output decline) and presence of biomarkers indicating structural damage are associated with marked increases in mortality in the appropriate clinical context, for example, in cases of critical illness in which they increase hospital mortality 3-7-fold ${ }^{6-9}$, whereas the same changes may not have long-term health implications in other settings, such as in marathon runners ${ }^{10}$. Given that the kidney provides life-sustaining functions, severe AKI can be lethal; hence, appropriate management including kidney replacement therapy (KRT), if needed, is essential.

In this Primer, we discuss the epidemiology of AKI in different economic settings, as well as the pathophysiology and diagnosis of AKI applied to a variety of settings, such as infections, sepsis, surgery, trauma, nephrotoxic medications, and heart disease, including its long-term consequences. Other causes of AKI or AKD, such as 
Table 1 | Criteria for defining AKI, AKD, CKD and NKD

\begin{tabular}{|c|c|c|c|c|}
\hline & AKI & AKD & CKD & NKD $^{*}$ \\
\hline Duration & $\leq 7$ days & $<3$ months & $>3$ months & NA \\
\hline $\begin{array}{l}\text { Functional } \\
\text { criteria }\end{array}$ & $\begin{array}{l}\text { Increase in } \mathrm{s} C r \text { by } \geq 50 \% \\
\text { within } 7 \text { days or increase } \\
\text { in } \mathrm{sCr} \text { by } \geq 0.3 \mathrm{mg} / \mathrm{dl} \\
(26.5 \mu \mathrm{mol} / \mathrm{l}) \text { within } \\
2 \text { days or oliguria for } \\
\geq 6 \text { hours }\end{array}$ & $\begin{array}{l}\text { AKI or GFR }<60 \mathrm{ml} / \mathrm{min} / \\
1.73 \mathrm{~m}^{2} \text { or decrease } \\
\text { in GFR by } \geq 35 \% \text { over } \\
\text { baseline or increase } \\
\text { in sCr by }>50 \% \text { over } \\
\text { baseline }\end{array}$ & $\begin{array}{l}\text { GFR }<60 \mathrm{ml} / \\
\mathrm{min} / 1.73 \mathrm{~m}^{2}\end{array}$ & $\begin{array}{l}\text { GFR } \geq 60 \mathrm{ml} / \mathrm{min} / 1.73 \mathrm{~m}^{2}, \\
\text { stable GFR (no decrease by } 35 \% \\
\text { within } 3 \text { months), stable sCr (no } \\
\text { increase by } 50 \% \text { within } 3 \text { months } \\
\text { or increase by } 0.3 \mathrm{mg} / \mathrm{dl} \text { within } \\
2 \text { days), no oliguria for } \geq 6 \text { hours }\end{array}$ \\
\hline AND/OR & OR & OR & OR & AND \\
\hline $\begin{array}{l}\text { Structural } \\
\text { criteria }\end{array}$ & Not defined & $\begin{array}{l}\text { Elevated marker } \\
\text { of kidney damage } \\
\text { (albuminuria, } \\
\text { haematuria or pyuria } \\
\text { are most common) }\end{array}$ & $\begin{array}{l}\text { Elevated } \\
\text { marker of } \\
\text { kidney damage } \\
\text { (albuminuria is } \\
\text { most common) }\end{array}$ & No marker of kidney damage \\
\hline
\end{tabular}

AKD, acute kidney diseases and disorders; AKI, acute kidney injury; CKD, chronic kidney disease; GFR, glomerular filtration rate; $\mathrm{NKD}$, no kidney diseases; $\mathrm{s} C r$, serum creatinine level. *NKD implies no functional or structural criteria according to the definitions for AKI, AKD or CKD.

Hepatorenal syndrome Impaired kidney perfusion and function in patients with advanced liver failure as a consequence of marked abnormalities in arterial and venous circulation, as well as overactivity of endogenous vasoactive systems. hepatorenal syndrome, glomerulonephritis, acute forms of glomerulonephritis or thrombotic microangiopathies (which can present as AKI), kidney transplantation or neonatal circumstances ${ }^{11-14}$, are not discussed in detail. We detail current approaches and cornerstones of AKI management, summarize how AKI and its long-term effects affect patients' quality of life and highlight ongoing and future initiatives to improve care for patients with this disorder.

\section{Epidemiology Incidence}

The global burden of AKI-related mortality exceeds by far that of breast cancer, heart failure or diabetes ${ }^{15}$, with mortality remaining high during the past 50 years. In general, the incidence of AKI is reported as either community-acquired or hospital-acquired AKI. In high-income countries (HIC), AKI is predominantly hospital-acquired, whereas community-acquired AKI is more common in lower-income settings ${ }^{15,16}$. These patterns apply to both adults and children globally. In HIC overall, patients with AKI tend to be older, have multiple comorbidities, and have access to dialysis and intensive care if needed ${ }^{17}$. Post-surgical or diagnostic interventions, or iatrogenic factors are the main causes of $\mathrm{AKI}$ in $\mathrm{HIC}^{18}$ (BOX 1). However, in low-income settings, numerous community-acquired causes exist, such as sepsis, volume depletion, toxins (bites, remedies) and pregnancy ${ }^{19}$. Patients tend to be younger than those in

\footnotetext{
Author addresses

${ }^{1}$ Center for Critical Care Nephrology, Department of Critical Care Medicine, University of Pittsburgh, Pittsburgh, PA, USA.

${ }^{2}$ Nephrology and Dialysis Unit, Meyer Children's University Hospital, Florence, Italy. ${ }^{3}$ Faculty of Medicine and Biomedical Sciences, Yaounde General Hospital, University of Yaounde, Yaounde, Cameroon.

${ }^{4}$ Department of Medicine, University of Padova, Padua, Italy.

${ }^{5}$ Department of Nephrology, Dialysis and Kidney Transplant, International Renal Research Institute, San Bortolo Hospital, Vicenza, Italy.

${ }^{6}$ Department of Anaesthesiology, Intensive Care and Pain Medicine, University Hospital Münster, Münster, Germany.

${ }^{7}$ Division of Nephrology, Department of Medicine IV, LMU University Hospital, LMU

Munich, Munich, Germany.
}

HIC, access to care is more challenging and women are under-represented in the patient population.

A meta-analysis of 154 studies that defined AKI according to the 2012 Kidney Disease Improving Global Outcomes (KDIGO) classification compiled data of $3,585,911$ people from mostly north of the Equator $(84 \%$ $\mathrm{HIC})$ and reported community-acquired AKI in $8.3 \%$ of ambulatory patients and in $20.0-31.7 \%$ of patients at various levels of in-hospital care ${ }^{20}$. Others report much lower incidences, which may relate to AKI definitions and local circumstances ${ }^{21}$. The average pooled mortality rate was $23 \%$ but reached $49.4 \%$ in those requiring $\mathrm{KRT}^{20}$.

In HIC, AKI is most prevalent in intensive care units (ICUs), where it occurs mostly in older patients in the context of multiorgan failure with high mortality. In this setting, AKI-related costs are very high, and prevention is difficult. The incidence of AKI in ICUs increased over the past decades in world regions with ageing population $\mathrm{s}^{20,22}$. In low- to middle-income countries (LMIC), AKI occurs mostly as a complication of a single disease with a pooled incidence and mortality rate of $21 \%$, respectively; however, the pooled mortality rises to $42 \%$ in those with KDIGO stage 3 and to $46 \%$ in those requiring $\mathrm{KRT}^{23}$. Around $77 \%$ of AKI in LMIC is community-acquired, with dehydration being the most common cause, whereas community-acquired AKI accounts for $50 \%$ in HIC, with hypotension and shock being the main causes ${ }^{24}$. AKI in LMIC is considered largely preventable with public health initiatives, which need to be cost-effective, as care for patients with severe stages of AKI quickly becomes unaffordable ${ }^{22}$. Globally, the mean age of patients with AKI is 60 years but this declines with decreasing socioeconomic status to 50 years in LMIC. Independent of the socioeconomic status of the region, $60 \%$ of patients with AKI are men ${ }^{24}$, which may either relate to the limited access of women to healthcare or to sex-related risks for AKI in males ${ }^{25}$. Few ethnic variations have been reported. In Asia, the risk of AKI after cardiac surgery was higher in India and Malaysia than in China $^{26}$. In the USA, the risk of pregnancy-related AKI was considerably higher in Black women than in white women ${ }^{27}$. Racial differences in the 


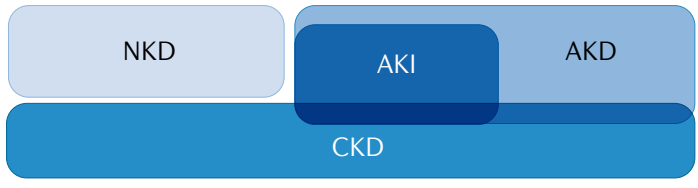

Fig. 1 | Relationship between AKI, AKD, CKD and NKD. Acute kidney injury (AKI) is a condition defined by kidney function markers (serum creatinine and urine output levels) and duration $\leq 7$ days, which is part of a group of acute kidney diseases and disorders (AKD). Chronic kidney disease (CKD) describes persistent ( $>3$ months) alteration of kidney function, and both $\mathrm{AKI}$ and $\mathrm{AKD}$ can occur in patients with or without precedent CKD. Patients with no kidney disease (NKD) do not overlap with any of these entities.

\section{Kidney failure}

Complete (and life-threatening) loss of kidney function; kidney failure replaces the term end-stage kidney disease in new nomenclature put forward by the KDIGO.

Glomerular filtration rate The volume of filtrate passing the glomerular filtration barrier per unit of time; a marker of excretory kidney function.

Third-space effusions

Accumulation of fluid in body cavities

Pulmonary congestion An accumulation of fluid in the lungs, resulting in impaired gas exchange and arterial hypoxaemia. incidence of AKI exist and may involve multiple factors. A multivariate analysis of a US study cohort identified socioeconomic status to account for the higher risk of AKI in African American individuals than in white individuals ${ }^{28}$.

\section{Risk factors}

Risk factors for AKI include environmental, socioeconomic and/or cultural factors, as well as factors related to the process of care, acute exposures and patients themselves. Environmental factors include inadequate drinking and waste water systems, insufficient control of infectious diseases and insufficient health care system ${ }^{23}$. Patient-related factors can be modifiable, for example, volume depletion, hypotension, anaemia, hypoxia and use of nephrotoxic drugs, or nonmodifiable, for example, chronic kidney, heart, liver or gastrointestinal disease, diabetes and severe infections and sepsis. Rarer causes include genetic predispositions to myoglobinuria, haemoglobinuria and urolithiasis ${ }^{23}$. Further important risk factors for AKI are severe diseases, acute infections, sepsis, malaria, severe trauma, hypovolaemia, old age, pre-existing $\mathrm{CKD}$, acute organ failures, major surgeries (including cardiac surgery), being in the ICU with exposure to nephrotoxic drugs and opportunistic infections, chemotherapy for leukaemia or cancer, delayed graft function upon kidney transplantation, autoimmune disorders with rapid progressive kidney injury, cholesterol

\section{Table 2 | Proposed new definitions of $\mathrm{AKI}^{5}$}

\begin{tabular}{|c|c|c|}
\hline Stage & Functional criteria & $\begin{array}{l}\text { Damage criteria } \\
\text { (biomarker) }\end{array}$ \\
\hline $1 S$ & $\begin{array}{l}\text { No change or sCr increase }<0.3 \mathrm{mg} / \mathrm{dl} \text { and no urinary } \\
\text { output criteria }\end{array}$ & + \\
\hline $1 \mathrm{~A}$ & \multirow{2}{*}{$\begin{array}{l}\text { Increase of } \mathrm{sCr} \text { by } \geq 0.3 \mathrm{mg} / \mathrm{dl} \text { for } \leq 48 \text { hours or } \geq 150 \% \text { for } \\
\leq 7 \text { days and/or urinary output }<0.5 \mathrm{ml} / \mathrm{kg} / \mathrm{h} \text { for }>6 \text { hours }\end{array}$} & - \\
\hline 1B & & + \\
\hline $2 \mathrm{~A}$ & \multirow{2}{*}{$\begin{array}{l}\text { Increase of } \mathrm{sCr} \text { by }>200 \% \text { and/or urinary output } \\
<0.5 \mathrm{ml} / \mathrm{kg} / \mathrm{h} \text { for }>12 \text { hours }\end{array}$} & - \\
\hline $2 \mathrm{~B}$ & & + \\
\hline $3 \mathrm{~A}$ & \multirow{2}{*}{$\begin{array}{l}\text { Increase of sCr by }>300 \% \text { ( } \geq 4.0 \mathrm{mg} / \mathrm{dl} \text { with an acute } \\
\text { increase of } \geq 0.5 \mathrm{mg} / \mathrm{dl} \text { ) and } / \text { or urinary output } \\
<0.3 \mathrm{ml} / \mathrm{kg} / \mathrm{h} \text { for }>24 \text { hours or anuria }>12 \text { hours } \\
\text { and/or acute kidney replacement therapy }\end{array}$} & - \\
\hline $3 \mathrm{~B}$ & & + \\
\hline
\end{tabular}

AKI, acute kidney injury; sCr, serum creatinine level. Adapted from REF. ${ }^{5}$, CC BY 4.0 (https:// creativecommons.org/licenses/by/4.0/). crystal embolism and urinary tract obstruction ${ }^{24}$. In HIC, despite severe AKI occurring more frequently in the context of hospital-related risk factors, such as major surgery, bleeding, septic shock or drug toxicity in older patients with multiple diseases, milder forms of AKI can also be community-acquired $(\mathrm{BOX} 1)^{16,18,29-31}$. By contrast, in LMIC, community-acquired AKI affects younger, previously healthy individuals, with a relatively high prevalence of sepsis, obstetric complications or animal venom as causes ${ }^{18,32}$. In these settings, AKI may also be caused by HIV infection, hantavirus infection, malaria or dengue disease, if their prevalence is high ${ }^{18,30}$. COVID-19 is a risk factor for AKI in all world regions (BOX 2).

\section{Mechanisms/pathophysiology Kidney physiology and kidney lifespan}

The kidneys maintain homeostasis of body fluids, electrolytes, osmolality and $\mathrm{pH}$, excrete metabolic waste products and secrete hormones and bioactive molecules. As AKI disrupts homeostasis, severe AKI is potentially lethal unless KRT maintains homeostasis until kidney function recovers. AKI in settings of multiorgan failure is frequently lethal despite $\mathrm{KRT}^{33}$.

The kidneys are composed of nephrons, small independent functional units with a glomerular part filtering fluid and small molecules from the blood and a single tubule that reabsorbs most filtered molecules and secretes metabolic waste products, concentrating the urine to 1-2 litres per day. The number of nephrons is set at birth and declines with age starting from around 25 years of age ${ }^{34}$. As metabolic activity also declines with age, healthy individuals at age 70 do well with only half of the original nephron number without adaptation $^{35}$. However, low nephron endowment at birth or any nephron loss beyond that of normal ageing shortens kidney lifespan; hence, the incidence of CKD and kidney failure ${ }^{36}$ requiring KRT increases in the elderly population ${ }^{37}$. AKI and CKD are connected because AKI can cause irreversible loss of nephrons at any phase of life and, therefore, shorten kidney lifespan (FIG. 2) ${ }^{38}$. Thus, $\mathrm{AKI}$ is an important risk factor for CKD, especially in ageing populations.

\section{Pathophysiology of kidney failure}

Loss of kidney excretory function implies disturbances in the main function of the kidneys (maintaining homeostasis), for example, through excretion of metabolic waste products. Serum creatinine and urea nitrogen levels are often used as biomarkers of reduced kidney function, but their use skews awareness towards the kidneys' excretory function ${ }^{1}$.

Fluid homeostasis is affected, as declining glomerular filtration rate (GFR) and activation of the reninangiotensin system promote fluid retention, which presents as peripheral oedema, third-space effusions, and pulmonary congestion, especially in those with heart failure (Supplementary Box 1) ${ }^{39}$. In addition, as urinary output determines potassium excretion, hyperkalaemia is a common complication of severe AKI. When hyperkalaemia leads to electrocardiogram changes, AKI constitutes a medical emergency and warrants immediate intervention. Both hyponatraemia and hypernatraemia 


\section{Box 1 Selected causes of AKI}

\section{Pre-renal causes, impaired renal perfusion}

Cardiorenal syndrome, including heart failure with reduced ejection fraction, right-sided heart failure and venous congestion

Shock, including haemorrhagic shock, hypovolaemic shock and septic shock Abdominal compartment syndrome

Kidney transplant, including delayed graft function

Medication, including angiotensin-converting enzyme inhibitors and angiotensin receptor blockers

\section{Intra-renal causes}

Thrombotic microangiopathies, cholesterol embolism, anti-glomerular basement membrane disease and immune complexes and anti-neutrophilic cytoplasmic autoantibody vasculitis

Sickle cell anaemia and sepsis

Systemic infections and sepsis, pyelonephritis, drug-related or heavy metal-related tubule necrosis, crystal-induced nephropathy (including crystals related to bile pigments, causing bile cast nephropathy), myoglobin (rhabdomyolysis), contrast media, light chains (monoclonal gammopathies) and metabolites (acute urate or oxalate nephropathy)

Acute cellular rejection, acute interstitial nephritis, immune checkpoint inhibitorrelated and cytokine release syndrome upon chimeric antigen receptor (CAR) T cell therapy

Post-renal causes, urinary tract obstruction

Bilateral ureteral obstruction, bladder dysfunction and urethral obstruction

$\mathrm{AKI}$, acute kidney injury.

\section{Fixed acid}

An acid that accumulates in the body as a result

of digestion, disease or

metabolism and cannot

be excreted by the lungs

(non-volatile or fixed).

\section{Anion gap}

The difference between the

sum of routinely measured

cations $\left(\mathrm{Na}^{+}\right.$and $\left.\mathrm{K}^{+}\right)$and the

sum of routinely measured

anions $\left(\mathrm{Cl}^{-}\right.$and $\left.\mathrm{HCO}_{3}^{-}\right)$

in serum.

\section{Azotaemia}

Abnormally high levels of nitrogen-containing

compounds in the blood, occurring when the kidneys are no longer able to excrete nitrogen waste products via the urine.

Venous congestion

A distention of veins filled with blood as a result of mechanical obstruction or right ventricular failure.

Acute tubular necrosis

A form of AKI that involves loss of entire tubule segments due to necrotic death of tubular epithelial cells. may occur when the kidney loses the capacity for urine concentration or dilution as needed. Impaired phosphate clearance leads to hyperphosphataemia.

AKI also affects acid-base homeostasis. A declining capacity for the excretion of fixed acids in patients with AKI causes tubular metabolic acidosis and respiratory compensation via an increased ventilatory drive ${ }^{40}$. Although a hyperchloraemic metabolic acidosis develops initially, widening of the anion gap is often seen as the result of accumulation of phosphate, sulfate and small organic anions in the bloodstream. A decline in the capacity to excrete metabolic waste products is indicated by azotaemia but implies disturbance of homeostasis of hundreds, if not thousands, of other metabolites that are not waste products, which all together account for symptoms of uraemia, such as fatigue, tremor or confusion.

Importantly, kidney failure affects most organ systems of the body (FIG. 3). Many of the AKI-related uraemic toxins originate from the intestinal microbiota such as indoxyl sulfate or p-cresyl sulfate. The microbiota itself undergoes shifts in its composition, owing to AKI and the accompanying acidosis, azotaemia, intestinal ischaemia, and other alterations of the intestinal microenvironments, which affects the microbiota's secretome and metabolites needed for normal human physiology $y^{41-43}$. The lungs are affected by hyperpnoea to compensate for metabolic acidosis, hypervolaemia, cytokines, oxidative stress and cytotoxic elements of necrotic cell debris (released by parenchymal necrosis in the kidneys, causing microvascular injury, and eventually acute respiratory distress syndrome $)^{44,45}$. AKI affects cardiac function via acidosis, hyperkalaemia, uraemic toxins, hypervolaemia, hypertension, and systemic inflammation ${ }^{46}$. Uraemic encephalopathy also involves systemic oxidative stress responses ${ }^{41,47}$.

\section{Kidney injury and recovery}

The term AKI, as defined, ascribes a decline in kidney excretory function and frequently (but not always) also tissue injury. Volume depletion, haemorrhagic shock, and heart failure with reduced ejection fraction, hepatorenal syndrome, venous congestion or hypercalcaemia can cause potentially reversible hypoperfusion of the kidney that transiently reduces GFR without parenchymal injury (BOX 1, FIG. 4), but as ischaemia persists ischaemic tubular injury may turn into tubule necrosis ${ }^{48}$. Nephrotoxic drugs and radiocontrast agents contribute to AKI in hospitalized patients and are also common causes of community-acquired $\mathrm{AKI}^{49,50}$. Multiple mechanisms are involved but most drugs can be classified into six main categories. First, some drugs, most notably chemotherapeutics (such as cisplatin) and antimicrobials (such as amphotericin or aminoglycosides), have direct chemical nephrotoxicity. Drugs that are cleared via the kidneys, such as vancomycin, are particularly problematic because drug-induced kidney dysfunction can lead to accumulation of the drug and its metabolites, further amplifying the toxicity ${ }^{51}$. Second, some agents are nephrotoxic via immune-mediated mechanisms, leading to allergic tubulointerstitial nephritis, which can be difficult to diagnose owing to a lack of overt signs ${ }^{52}$. Third, some drugs, most notably angiotensin-converting enzyme (ACE) inhibitors and angiotensin receptor blockers, can cause a decrease in GFR by affecting intrarenal haemodynamics ${ }^{53}$. Drugs with haemodynamic effects on kidney perfusion can protect nephrons from hyperfiltration-related progression to CKD, although a substantial and persistent decline of kidney perfusion may lead to ischaemic acute tubular necrosis (ATN $)^{54,55}$. Fourth, when drug metabolites crystallize inside the kidney tubules, they can cause intrarenal obstruction of urinary flow and kidney injury ${ }^{56}$. Fifth, the mechanism of action of some drugs can contribute to AKI, such as failure intrarenal haemorrhage associated with oral anticoagulants or acute urate nephropathy associated with uricosuric drugs. Finally, the renal excretion of some drugs or drug metabolites competes with creatinine at the same tubular transporter, mimicking AKI, although other functions of the kidney remain unaffected.

Many other triggers can cause ATN (BOX 1), but combinations of triggers are common, especially in ICU settings. Human data are scarce but animal models suggest that ATN is not a passive process but instead involves different forms of regulated necrosis, such as necroptosis and ferroptosis, that can synchronize tubular cell death along entire tubule segments and spares glomeruli ${ }^{57,58}$. The necrotic tubular cells can form casts and obstruct the lumen of tubules ${ }^{59}$. Tubule necrosis involves the release of danger signals that activate Toll-like and other pattern recognition receptors on resident immune cells in the kidney interstitium, namely $\mathrm{MHCII}^{+} \mathrm{F} 4 / 80^{\mathrm{Hi}}$ conventional dendritic cells in mice ${ }^{60}$; however, little is known about the immune cell dynamics in human AKI. Activation of these cells triggers the influx of neutrophils in the early injury phase and M1 macrophages and other myeloid cells in the late injury phase, which all contribute to a local inflammatory response that accelerates 


\section{Box 2 | AKI during COVID-19}

The coronavirus disease 2019 (COVID-19) pandemic has evolved as a new trigger of acute kidney injury (AKI). Risk factors for AKI on admission include: the level of viraemia; respiratory compromise; organ involvement other than lungs; leukocytosis and lymphopenia; high levels of C-reactive protein, ferritin and D-dimers; and hypovolaemia and dehydration or rhabdomyolysis. Almost half of patients hospitalized for COVID-19 experience AKI similar to high-risk AKI settings, such as cardiac surgery or sepsis ${ }^{200}$. One study reported that $39 \%$ of these patients develop AKI stage $1,19 \%$ develop stage 2 , and $42 \%$ develop stage $3^{200}$. The time of AKI matched the time of intubation in most patients ${ }^{201}$. Older age, hypertension, smoking, obesity, diabetes, cardiovascular disease or congestive heart failure, precedent CKD, use of immunosuppressant drugs and, potentially, genetic variants predisposing to kidney disease (APOL1 and ACE2 polymorphisms) are other predisposing factors ${ }^{202}$. AKI is strongly associated with COVID-19 mortality ${ }^{200}$, whereas COVID-19 mortality is low without AKI ${ }^{203}$. COVID-19 may lead to kidney failure via different processes, including systemic immune and inflammatory responses induced by viral infection, systemic tissue hypoxia, local immunothrombosis causing hypoxia, reduced kidney perfusion, endothelial damage and direct epithelial infection with SARS-CoV-2 ${ }^{204-206}$. Interestingly, some but not all autopsy studies demonstrate that the presence of SARS-CoV-2 copies and proteins inside the kidneys is tightly associated with AKI and an increased COVID-19 mortality, especially in older patients ${ }^{206,207}$. However, the identification of viral particles inside kidney cells by electron microscopy is challenging ${ }^{208}$. AKI-related fluid, acid-base and metabolic disturbances may affect antiviral immunity and the resolution of inflammation. Thus, there is a strong rationale to target AKI for therapy in COVID-19 ${ }^{209}$. For example, beyond replacing kidney function, extracorporeal therapies also support other organs and achieve immunomodulation ${ }^{209}$. Among those that survive COVID-19-related AKI requiring dialysis, one in six patients remains dialysis-dependent at 60 days ${ }^{210}$.

tubule necrosis, an auto-amplification loop referred to as necroinflammation ${ }^{61,62}$. Unless the nephrotoxic trigger persists, as in persistent ischaemia, toxin exposure or alloimmunity, numerous counter-regulators support a resolution of necroinflammation, which is a requirement to launch healing responses.

The capacity to replace lost tubular cells is restricted to a subset of immature tubular cells (renal progenitors) scattered along the nephron that retain the capacity to replace single epithelial cells and, in some instances, to regenerate entire tubule segments ${ }^{63-65}$. Complete loss of these progenitors disrupts regeneration of an affected tubule segment, which can result in irreversible loss of the nephron (FIG. 4). The number of irreversibly lost nephrons in an AKI episode determines the long-term prognosis of kidney function ${ }^{63}$.

Nephron loss is difficult to appreciate in clinical practice because, even after persistent AKD, kidney function frequently recovers to some extent ${ }^{2,66,67}$. However, recovery of kidney function does not necessarily represent regeneration, as functional capacity can be augmented by polyploidization of tubular epithelial cells in unaffected nephrons (compensatory hypertrophy) ${ }^{63}$. This evolutionarily conserved mechanism enables life-saving increased functional outputs of differentiated parenchymal cells in organs with limited cell turnover. In the injured kidneys, processes such as dedifferentiation and mitotic events during which cells no longer contribute to functional performance would be incompatible with maintaining kidney function in the phase where this is most needed to assure survival ${ }^{68}$. However, in severe forms of ATN, polyploidization and progenitor proliferation cannot sustain a sufficient residual function, and severe ATN is lethal unless KRT is used to cover the time until a life-sustaining level of kidney function recovers.

\section{Long-term consequences of AKI or AKD}

The adaptations that occur in response to the irreversible loss of kidney cells or entire nephrons may ensure short-term survival but they have considerable trade-offs that affect long-term outcomes after an AKI or AKD episode (FIG. 4) (8-70. $^{68}$.

Irreversible nephron loss, fibrosis and CKD. Depending on the severity of AKI, few, many or most nephrons remain irreversibly destroyed and lost, which implies post-AKI CKD and a reduction of kidney lifespan ${ }^{66,71}$. Albuminuria following AKI is a clinical indicator of CKD, even if GFR seems fully recovered ${ }^{72}$. The effect of AKI on kidney lifespan is most evident in older adults in whom AKI-related nephron loss adds to age-related

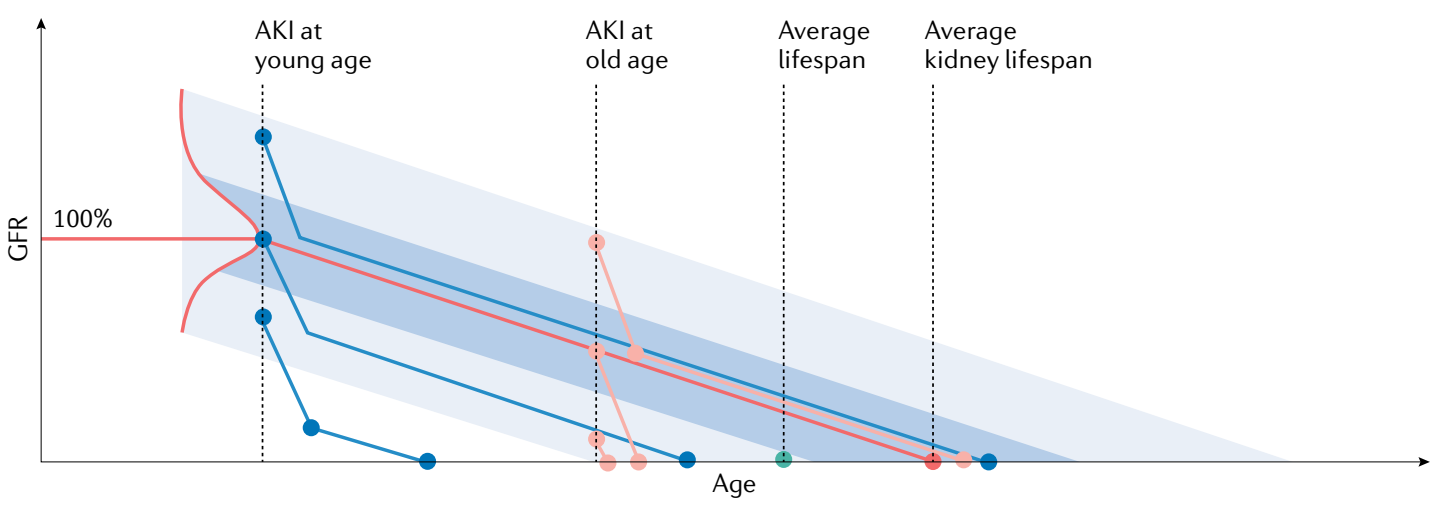

Fig. 2 | Consequences of AKI on kidney lifespan. Kidney lifespan depends on the individual endowment with nephrons, which follows a Gaussian curve at each given age (red line). The decline of nephron number and glomerular filtration rate (GFR) with age can be expressed as declining percentiles (blue shading). The average kidney lifespan exceeds human average lifespan. Each irreversible nephron loss that is related to an acute kidney injury (AKI) episode reduces kidney lifespan (sudden drop of solid lines); however, for those with high nephron endowment, this may not have consequences on kidney lifespan during their lifetime. However, for those with an average or, in particular, for those with low nephron endowment, the shortening of kidney lifespan implies the occurrence of kidney failure many years earlier — at worst directly after the AKI episode. Severe AKI at an older age (pink lines) has a more immediate effect on the remaining kidney lifespan than $\mathrm{AKI}$ at a younger age (blue lines). 
nephron loss and, frequently, to precedent CKD owing to previous injuries or chronic nephropathies, referred to as $\mathrm{AKI}$ on $\mathrm{CKD}^{73}$. Thus, as ATN implies nephron loss, the severity of ATN determines the effect on kidney lifespan ${ }^{66}$. In extreme cases, severe ATN can result in kidney failure with a need for persistent KRT.

Hypertension, cardiovascular disease and stroke. Survivors of AKI may face hypertension, which can be a sign of subclinical CKD. A retrospective cohort study identified a $22 \%$ increase in the risk of presenting with a blood pressure of $>140 / 90 \mathrm{mmHg}$ in those who had AKI compared with individuals who did not experience AKI after adjustments for demographic factors, precedent health status and cardiovascular risk factors ${ }^{74}$. Post-AKI $\mathrm{CKD}$ is associated with increased cardiovascular and cerebrovascular morbidity and mortality ${ }^{70,75,76}$. A metaanalysis including 254,150 adults $(55,150$ with AKI) found that $\mathrm{AKI}$ increased the risk of subsequent heart

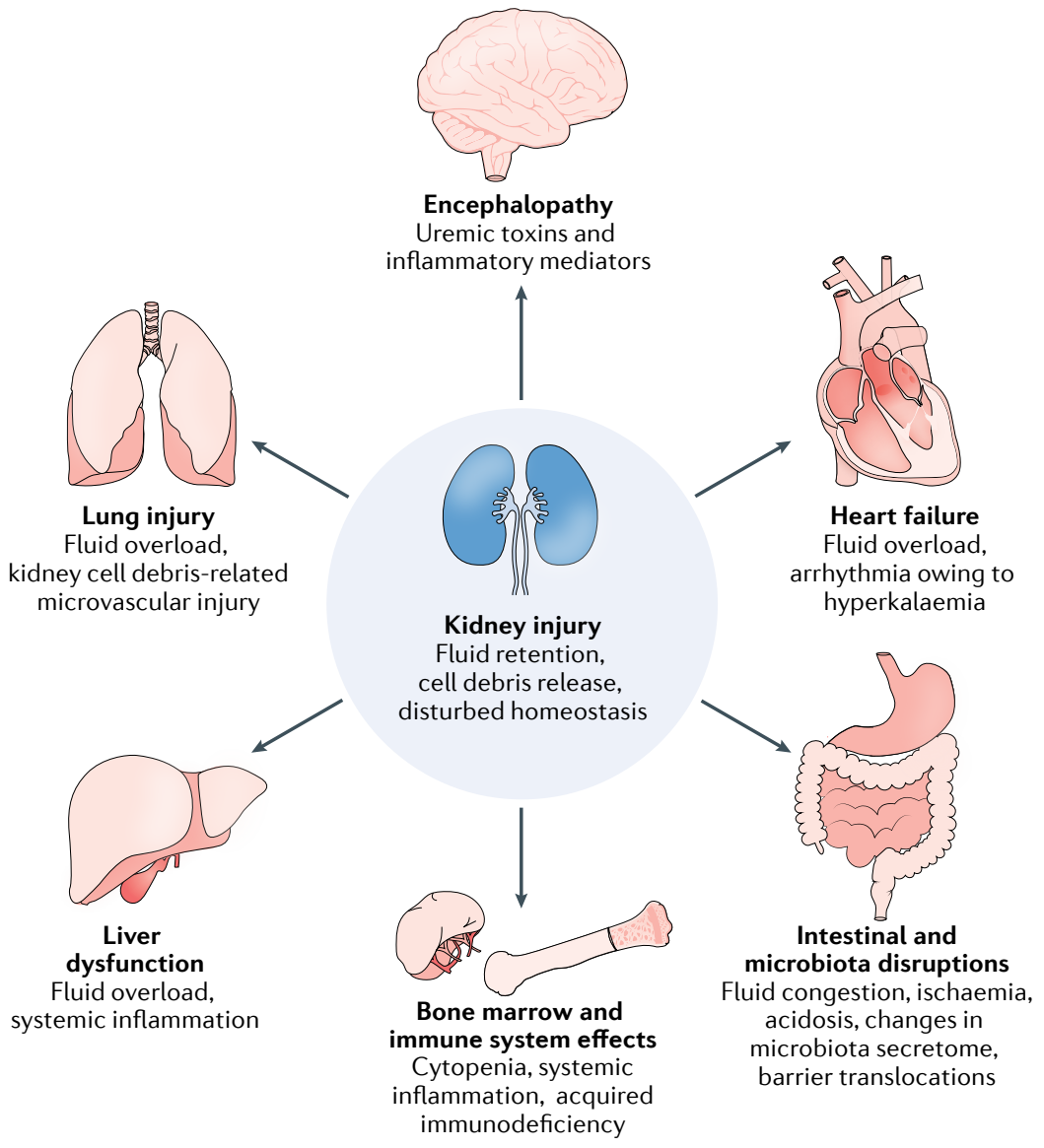

Fig. 3 | Systemic consequences of AKI. The kidneys maintain homeostasis; hence, acute kidney injury (AKI) affects almost all systems of the body, albeit in different ways. Fluid retention affects especially the lungs and the heart, frequently with clinical signs of respiratory or circulatory failure. Fluid retention also compromises the gastrointestinal system, for example the liver or the intestine, promoting intestinal barrier dysfunction and translocation of bacteria and bacterial toxins. Impaired uraemic toxin excretion affects the function of the brain, the heart, the bone marrow and the immune system, leading to neurocognitive defects, anaemia and acquired immunodeficiency accompanied by persistent systemic inflammation. Kidney cell necrosis releases debris into the venous circulation, which accumulates in the lungs and causes direct microvascular injury, thrombosis and, sometimes, acute respiratory distress syndrome. Adapted with permission from REF.199, Elsevier. failure by $58 \%$, myocardial infarction by $40 \%$ and stroke by $15 \%$. Whether this increase relates to an effect on the cardiovascular system during the AKI episode or to the increase in risk owing to post-AKI CKD is not clear.

Mortality. Survivors of AKI face an increased posthospitalization mortality. A meta-analysis of 110 studies that used the KDIGO definition of AKI found an AKIrelated mortality of $23 \%{ }^{20}$. In an analysis of $>5$ million patients discharged from hospital, mortality within 90 days was $35 \%$ in those with AKI and $13 \%$ in those without $\mathrm{AKI}^{78}$. Longer-term mortality among patients with AKI may also be increased. In a study in patients who underwent cardiac surgery, increase in mortality risk was independent of kidney function recovery at the time of hospital discharge and started only 4-5 years after surgery ${ }^{79}$. A systematic review including 47,017 patients who had been discharged from hospital reported 8.9 deaths per 100 person-years in those who had AKI compared with 4.3 deaths per 100 patient-years in those without $\mathrm{AKI}^{80}$. The most common causes of death after a hospitalization with AKI are cardiovascular disease (28\%) and cancer (28\%), with respective standardized mortality ratios nearly six-fold and eight-fold higher than those in the general population ${ }^{70}$. Cancers were mostly haematological and genitourinary.

An AKI episode implies a risk for subsequent development of kidney cancer and an AKI episode following partial nephrectomy for kidney cancer increases the risk of cancer reoccurrence ${ }^{81}$, probably because kidney injury induces DNA damage and a clonal expansion of mutated cells during the repair phase ${ }^{82}$. Indeed, renal progenitors that confer tubule regeneration upon ischaemic ATN can turn into tumour stem cells and set off monoclonal lesions in a papillary renal cell adenoma-carcinoma sequence $^{81,83}$. Kidney injuries to other nephron segments cause other types of kidney cancer ${ }^{81}$. Thus, kidney cancer is a long-term trade-off for kidney regeneration that supports short-term survival during ATN ${ }^{68}$.

\section{Diagnosis, screening and prevention}

Unlike myocardial infarction and other acute organ failures, AKI does not present with immediate onset of alarming symptoms such as chest pain, dyspnoea, palsy or blindness; hence, diagnosis requires specific technical assessments. The best overall index of kidney function is the GFR, but direct GFR measurement is difficult. Usually, the GFR is estimated by using serum levels of endogenous filtration markers, such as creatinine. Several studies have demonstrated that small increases of serum creatinine are associated with worse outcomes of $\mathrm{AKI}^{84,85}$. In addition, urine output is a sensitive parameter of kidney function and a biomarker of tubular injury ${ }^{86}$. However, the relationship between urine output, GFR and tubular injury is very complex.

\section{Diagnostic and classification criteria}

Adults. Evidence exists that suggests that an acute and small impairment of kidney function, manifested by changes of blood chemistry and urine output, is associated with a worse outcome of $\mathrm{AKI}^{84,85,87}$. In contrast to 


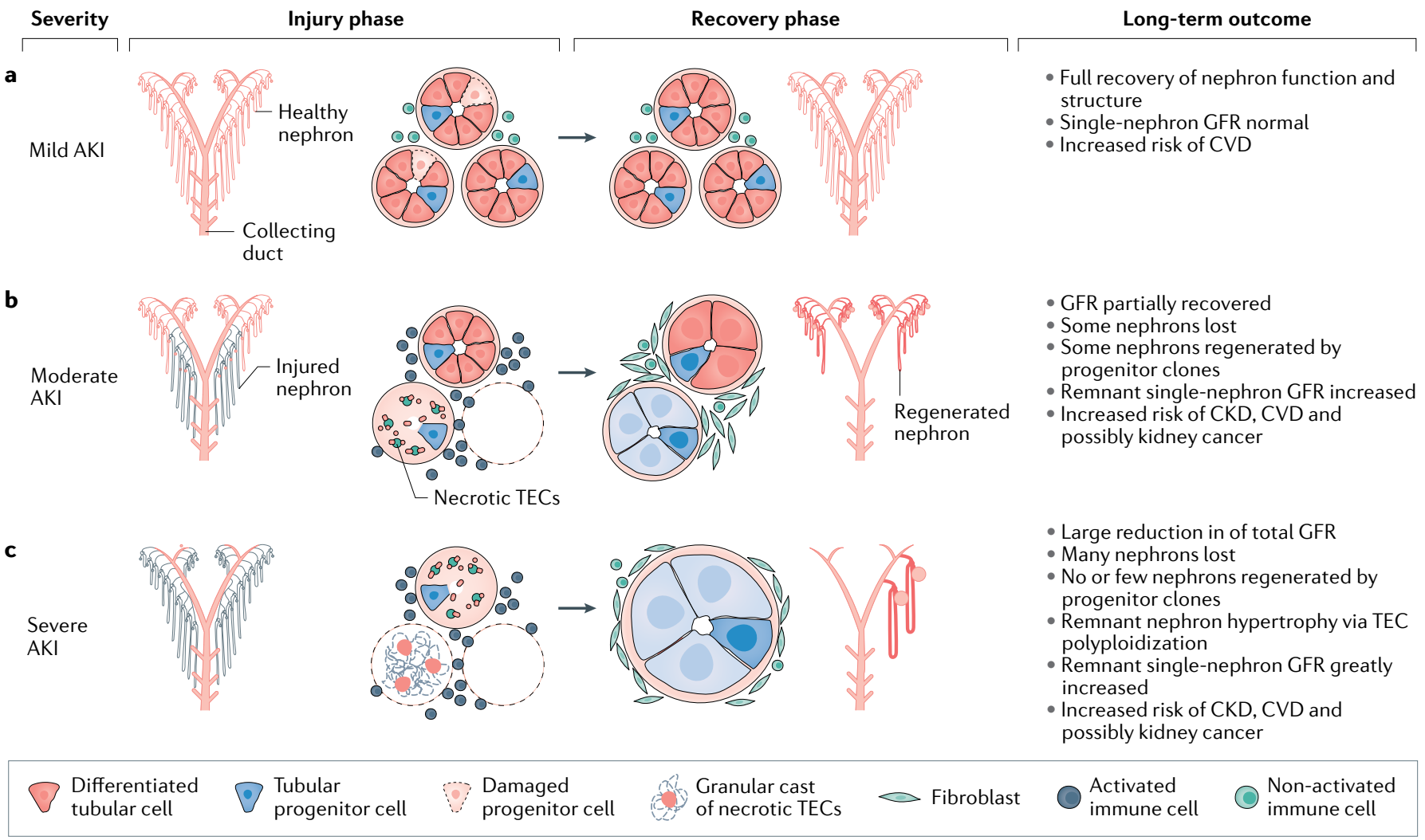

Fig. 4 | Main principles of the pathophysiology of AKI. a | Mild acute kidney injury (AKI), defined by a transient decline in urinary output or excretory function, involves no or minimal kidney cell necrosis or loss. Precedent and subsequent nephron numbers remain identical and no persistent adaptive cellular responses are necessary. In the long term, the risk of cardiovascular disease (CVD) is somewhat increased, which may also depend on the underlying cause of AKI. $\mathbf{b}$ | Whenever AKI is associated with kidney cell or tubule necrosis, the affected cells are irreversibly lost during the phase of acute necroinflammation, as indicated by activated immune cells in the interstitial compartment. Renal progenitor cells are more resistant to death and their clonal expansion may facilitate the structural and functional recovery of some injured nephrons. Nephrons in which injured segments do not recover undergo atrophy, are irreversibly lost and are replaced by fibrous tissue that stabilizes the structural integrity of remnant nephrons. Resulting hyperfiltration requires an increase of the functional capacity of remnant nephrons achieved through an increase in their dimensions, with tubular epithelial cells (TECs) undergoing polyploidization, indicated by an increased size of cytoplasm and cell nuclei. Depending on the number of remnant nephrons, their capacity for adaption (kidney reserve), and filtration load (dependent on body weight, fluid intake, diet and others), glomerular filtration rate (GFR) can return to baseline. This status already qualifies as CKD, even if GFR returns to baseline. The adaptive changes of CKD imply a higher risk of CVD and possibly kidney cancer, and the irreversible loss of nephrons reduces kidney lifespan. c | When severe AKI involves extensive tubule necrosis, the consequences on nephron number are substantial. Tubule recovery occurs only in those nephrons with surviving progenitor cells. Adaptation to filtration and metabolic demands results in large increases in the dimensions of the few surviving nephrons (megalonephrons). Such adaptations frequently exceed the adaptive capacity of podocytes, leading to secondary focal segmental glomerulosclerosis and subsequent loss of the remnant nephrons (that is, progressive CKD). Cellular adaptation-related polyploidization and senescence, as well as nephron loss-related scarring, drives interstitial fibrosis and progressive kidney atrophy. These adaptive changes strongly increase the risk of CVD and possibly kidney cancer. Kidney lifespan is drastically reduced and some patients remain on kidney replacement therapy.
Fluid resuscitation

Large-volume intravenous fluid replacement to treat $\mathrm{AKI}$ and circulatory shock due to severe intravascular volume depletion. the old term acute renal failure, the Risk, Injury, Failure, Loss of kidney function, and End-stage kidney disease (RIFLE) and Acute Kidney Injury Network (AKIN) classifications provided updated definitions of AKI that encompass the complete spectrum of the syndrome from small increases of serum creatinine to requirement of KRT. The RIFLE and AKIN classifications have three severity grades based on changes of serum creatinine level or urine output, and the worse of these two criteria is used to define the grade. RIFLE and AKIN thus introduced a conceptual framework for how to diagnose and stage AKI, but further modifications were needed to meet the clinical complexity of AKI, especially outside ICUs or hospital care.

The 2012 KDIGO guideline defined the diagnostic criteria for $\mathrm{AKI}$ and $\mathrm{AKD}^{1}$. Unlike earlier recommendations, the KDIGO criteria no longer require adequate fluid resuscitation to be performed and urinary obstruction excluded before using the criteria. In particular, patients with CKD are predisposed to develop AKI, because $\mathrm{CKD}$ is an independent risk factor for $\mathrm{AKI}^{88-92}$. However, the diagnosis of AKI in patients with CKD is difficult, because these patients have impaired kidney function and the percentage changes in serum creatinine level after AKI are in part confounded by baseline kidney function ${ }^{93}$. Only larger absolute rises in creatinine levels indicate an independent association with mortality ${ }^{94}$. However, AKI in patients with CKD conveys serious risks, which is acknowledged by defining any rise of serum creatinine to $>4 \mathrm{mg} / \mathrm{dl}$ as AKI stage $3^{1}$.

The KDIGO criteria use a decline in urine output but reducing urinary output is also a physiological 


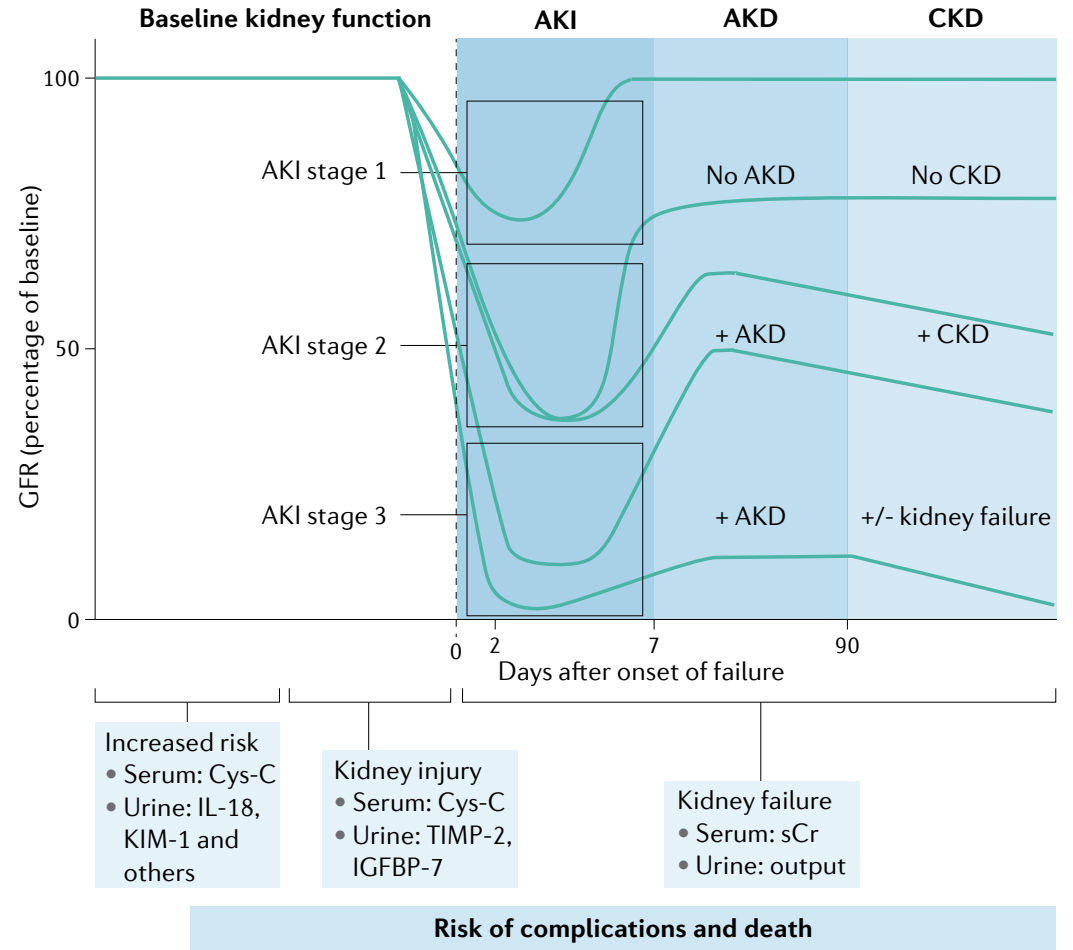

Fig. 5 | Severity of AKI and long-term kidney outcome. Certain biomarkers indicate early kidney injury or subclinical acute kidney injury (AKI) as a risk factor for proceeding to AKI according to the Kidney Disease Improving Global Outcomes (KDIGO) definitions. AKI itself is indicated by injury markers in blood and urine before any impairment of kidney function (as measured by serum creatinine levels and urine output) occurs. The three stages of AKI are defined by the extent of renal function impairment. Patients with $\mathrm{AKI}$ in which structural damage causing irreversible nephron loss does not occur may fully recover. AKI with structural damage frequently lasts $>7$ days, which is classified as acute kidney disease (AKD), and the irreversible nephron loss precludes restoration of the baseline glomerular filtration rate (GFR), resulting in chronic kidney disease (CKD) or persistent kidney failure. Cys-C, cystatin C; IGFBP-7, insulin-like growth factor-binding protein 7; IL-18, interleukin 18; KIM-1, kidney injury molecule 1 ; $\mathrm{sCr}$, serum creatinine level; TIMP-2, metalloproteinase inhibitor 2.

mechanism in response to reduced fluid intake or fluid losses, for example. by sweating, which readily responds to fluid intake and usually does not imply tubule injury. Injured tubules no longer respond to diuretics owing to the loss of the required sodium transporters; hence, a single bolus of a loop diuretic not followed by a substantial increase of urinary output, referred to as the furosemide stress test, indicates tubule injury ${ }^{95}$. In fact, the occurrence of AKI is considerably higher with abnormal urine output and serum creatinine levels compared with abnormal serum creatinine levels alone $(62.1 \%$ versus $17.7 \%)^{96,97}$.

Children. The different AKI definitions were developed for average-sized adults. After 2 years of age, kidney growth results in a GFR equivalent to that of adults when adjusted for body surface area ${ }^{98,99}$, but the serum creatinine criterion is difficult to apply to smaller paediatric patients, because they have a lower muscle mass that does not achieve high serum creatinine values. Thus, the paediatric RIFLE (pRIFLE) criteria were developed, which stage AKI by the rise in creatinine levels, fall in GFR, or decrease in urine output ${ }^{100,101}$ The KDIGO guidelines ${ }^{1}$ also refer to the pRIFLE criteria for the definition of AKI in children. However, the definition should only be used in children $>1$ month of age. The practical value of pRIFLE was shown in a trial including 103 patients (median age 4.5 years) ${ }^{102}$.

\section{Screening and risk assessment with biomarkers}

Around half of the patients with stage 1 AKI have elevated biomarkers and histologic abnormalities on kidney biopsy, whereas most of the patients with stage 3 AKI have both ${ }^{96,103}$. Serum creatinine levels and urine output are two functional biomarkers that have several limitations ${ }^{1}$. Urine output has a low specificity because this parameter can be influenced by several factors, including hypovolaemia and the use of diuretics. By contrast, serum creatinine level has low sensitivity in previously healthy kidneys, because the serum creatinine levels increase only if at least $50 \%$ of the functional nephrons are lost. In patients with low baseline GFR, minor changes in kidney function can already meet the threshold of an increase of serum creatinine of $0.3 \mathrm{mg} / \mathrm{dl}$, that is, AKI.

Novel biomarkers were not included in the 2012 KDIGO guidelines ${ }^{1}$. AKI biomarkers indicate different aspects of AKI and can be broadly divided into functional or damage biomarkers (Supplementary Box 2) 104-106. $^{10}$. Although biomarkers, such as IL-18 or kidney injury molecule 1 (KIM-1), are available and most of them have a very good predictive value, limitations exist, including poor predictive performance when the timing of the kidney insult is unknown; hence, they are implemented only inconsistently in clinical practice ${ }^{107-109}$.

In contrast to functional biomarkers, damage biomarkers might be able to identify patients at high risk for AKI (FIG. 5). However, these damage biomarkers should not be used in all patients regardless of their AKI risk profile, because their sensitivity would be low. The concept of renal angina, analogous to troponin measurement in patients with coronary angina, was introduced in 2010 and recommends that biomarkers should be measured only in patients at risk and with a specific clinical condition (for example, sepsis or major surgery ${ }^{110}$. Measuring damage biomarkers in these patients substantially improves their positive predictive value ${ }^{110}$.

Different biomarkers relate to various pathophysiological processes mediating $\mathrm{AKI}^{5}$ and might help in detecting AKI earlier. These markers will be critical in developing targeted therapies and designing clinical trials for patients with AKI. Four biomarkers that can be measured at the bedside by using point-of-care devices may be particularly useful in daily practice. Cystatin C levels are probably redundant to creatinine levels. Several neutrophil-gelatinase-associated lipocalin (NGAL) isoforms are released by the kidney and by immune cells ${ }^{111}$. In the urine of healthy individuals, the concentration of NGAL is very low. After an insult, NGAL plasma and urine levels increase considerably, suggesting a role of NGAL for the kidneys that is analogous to that of troponin for the heart ${ }^{112}$. NGAL has been shown to have very high sensitivity and specificity for predicting AKI in children undergoing congenital heart surgery ${ }^{113}$. However, other studies in patients with different 
G1 phase

The G1 phase is the first of four cell cycle phases that takes place in eukaryotic cell division and describes the period from the end of cell division to the beginning of DNA replication. comorbidities found a limited predictive performance of NGAL, potentially because immune cell-derived NGAL may not necessarily imply $\mathrm{AKI}^{104,114}$. The explanations for these contradictory results include poor performance of the standard markers of urine output and serum creatinine levels, preexisting comorbidities and timing of biomarker assessment, because NGAL has a good predictive value only in patients with previously normal kidney function ${ }^{115}$. Thus, NGAL can be used in patients with normal kidney function and a well described insult and in patients with precedent $\mathrm{CKD}^{116,117}$.

Unbiased screening for urinary biomarkers that can predict subsequent AKI revealed cell cycle arrest markers as top candidates ${ }^{118}$. Indeed, cell cycle arrest of kidney tubular epithelial cells is involved in the pathogenesis of $\mathrm{AKI}^{119}$. However, the predictive performance was only moderate in some studies ${ }^{120,121}$. Once the kidney experiences stress, tubular epithelial cells arrest in the G1 phase to avoid and recover from damage ${ }^{122}$. As cell stress is one of the first events during AKI, metalloproteinase inhibitor 2 (TIMP2) and insulin-like growth factor-binding protein 7 (IGFBP7) are detectable in urine very early during development of $\mathrm{AKI}^{118,123}$. Several trials have shown that urinary TIMP2 and IGFBP7 levels predict the development of AKI, kidney recovery and mortality ${ }^{18,123-125}$. In a multicentre observational trial in critically ill patients, combined TIMP2 and IGFBP7 measurement showed very good performance in predicting moderate to severe AKI (area under the curve (AUC) of 0.80), and considerably improved risk stratification when added to a clinical model ${ }^{126,127}$. In addition, TIMP2 and IGFBP7 showed very good predictive value in diagnosing $\mathrm{AKI}$ associated with cardiac surgery ${ }^{123}$. Of note, these biomarkers can also be used to predict adverse long-term outcomes, because their early measurement in the setting of critical illness may identify patients with AKI at increased risk of death or KRT in the following 9 months ${ }^{125}$. Further studies demonstrated an additional benefit of using these biomarkers in conjunction with the functional criteria of serum creatinine and urine output, as their combination improves the prediction of worse outcomes ${ }^{126,127}$.

Importantly, kidney damage without any loss of function, that is subclinical AKI, also affects outcomes ${ }^{128,129}$. Thus, the Acute Disease Quality Initiative (ADQI) group proposed an extended definition of AKI, which includes functional and damage biomarkers (TABLE 2) . Before using this new definition in daily clinical practice, further research is needed to evaluate whether elevation in biomarkers without any changes in urine output or serum creatinine is associated with worse kidney and patient outcomes.

\section{Other methods of measuring kidney function}

To date, no validated method exists for continuously measuring GFR in critically ill patients. However, one study published in 1994 demonstrated that such monitoring is feasible $\mathrm{e}^{130,131}$. Ongoing attempts to overcome practical obstacles may eventually implement this method in clinical practice ${ }^{130}$. Rapid kidney function assessment can be achieved by collecting urine and a repeated blood sample to determine creatinine clearance, which can also be reliably performed in the case of oliguria ${ }^{132,133}$.
Creatinine clearance has been shown to diagnose impairment of kidney function earlier than plasma creatinine rise $\mathrm{e}^{133}$.

\section{Prevention}

In LMIC, preventing volume depletion alone is believed to already have a major effect on the incidence of $\mathrm{AKI}^{23,134}$. Apart from preventing volume depletion and avoiding nephrotoxin exposure or overdosing, new biomarkers can identify patients at high risk of AKI (Supplementary Box 2). This approach can be used to stratify patient populations and implement different measures to prevent the development of AKI. Implementation of the 'KDIGO bundle' - consisting of optimization of volume status and haemodynamics, avoidance of nephrotoxic drugs, and prevention of hyperglycaemia, in patients at high risk of AKI as identified by biomarkers - can prevent AKI after cardiac surgery ${ }^{135}$. In a quality initiative programme, the implementation of supportive measures in biomarker-positive patients reduced the rate of moderate and severe AKI after cardiac surgery ${ }^{136}$, and the occurrence of AKI in patients undergoing abdominal surger $y^{137}$. Despite these data, only about $5 \%$ of high-risk patients receive these supportive measures ${ }^{138}$.

\section{Management}

Management of AKI is often not optimal. An audit in the UK in 2009 found that $>50 \%$ of patients were managed poorly, and $43 \%$ of AKI cases were recognized late or not at all ${ }^{8}$. Alerts in the electronic medical record have shown limited, if any, success ${ }^{9}$. However, when large enough sample sizes are considered, electronic alerts have measurable effects on the duration of hospitalization and on survival ${ }^{139}$. Because AKI is not a disease but rather a loose collection of syndromes, the first step in managing AKI is to determine its cause and recognizing prerenal causes (hypovolaemia) or postrenal causes (outflow obstruction) (BOX 1). Further work-up will be influenced by the patient's clinical context, location and history. In addition to identification and treatment of the likely AKI causes, general management considerations apply.

\section{Volume status}

Volume depletion itself impairs kidney function but does not damage the kidney unless it is severe and sustained (FIG. 6) ${ }^{2}$. However, volume depletion may contribute to various causes of AKI and attention to fluid status is a cornerstone of therapy ${ }^{140}$. Patients presenting with AKI from the community can be volume depleted, as can patients in hospital who receive diuretics or experience fluid losses from wounds or drains. Severe dehydration should never occur in a patient in hospital; however, remedying dehydration by providing inappropriate amounts of fluids without proper assessment of patients with AKI can result in fluid overload, which can have considerable harmful effects ${ }^{39}$. Patients needing intravenous fluid resuscitation should be under direct supervision of a physician and treatment benefits from guidance by haemodynamic monitoring ${ }^{39}$. Furthermore, a sudden need for fluid resuscitation requires a work-up to determine its cause (for example, occult haemorrhage 


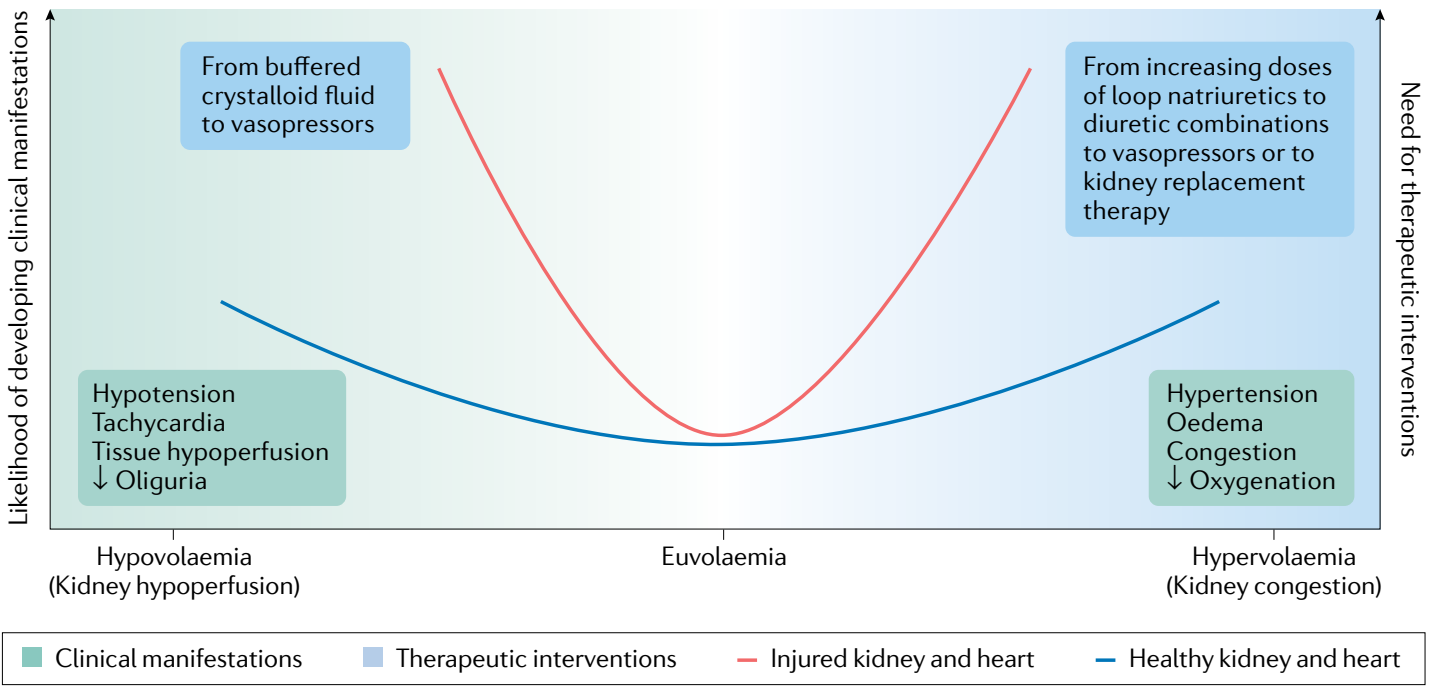

Fig. 6 | Fluid management in acute kidney injury. Both hypovolaemia (kidney hypoperfusion) and hypervolaemia (kidney congestion) compromise kidney function. Impaired cardiac function adds to both problems as renal and cardiac dysfunction aggravate each other, referred to as cardiorenal syndromes. An injured kidney or heart increases the likelihood of developing clinical symptoms of hypovolaemia or hypervolaemia compared with healthy organs. An increasing severity of symptoms requires escalating therapeutic interventions. Hypotension frequently indicates kidney hypoperfusion despite clinically apparent hypervolaemia whenever fluid redistributes to the venous system, into tissue interstitium or third compartments, for example, in hepatorenal syndrome, congestive heart failure or capillary leakage during sepsis. Kidney and/or heart failure drastically decrease both organs' capacity to maintain function during hypovolaemia or hypervolaemia. In an apparently euvolaemic patient with AKI, a single bolus of buffered crystalloid fluid can indicate the presence of subclinical hypovolaemia and hypoperfusion of the kidney (prerenal AKI). Prolonged administration of balanced crystalloids should be handled with caution in order not to promote oedema or congestion and not to decrease tissue oxygenation. Patients with hypervolaemia should not receive fluids for AKI but loop natriuretics. In patients who are critically ill with suppressed vasomotor response, vasopressors are frequently needed to improve cardiac output.

or sepsis). Of note, patients can develop oliguria from AKI and then develop fluid overload by injudicious administration of intravenous fluids in addition to the fluids they receive for medications and through nutritional support ${ }^{141}$. Importantly, fluid overload has been identified as an important cause of AKI, because venous congestion can compromise perfusion and cause direct damage to the kidney parenchyma.

\section{Haemodynamic management}

Management of blood pressure and cardiac function in the setting of conditions in which AKI arises (for example, septic shock or cardiac surgery) is complex and includes context-specific considerations, depending on the type of circulatory shock the patient experiences ${ }^{140,142}$. However, some general principles apply to haemodynamic management in patients with AKI (FIG. 6). In normal conditions, organs, including the kidneys, are adequately perfused at a mean arterial pressure (MAP) of $65 \mathrm{mmHg}^{143}$. Studies investigating whether increased MAP targets should be used for patients in the ICU had mixed results ${ }^{143-145}$. Patients with severe (and perhaps poorly controlled) hypertension may benefit from a higher MAP when in shock, but no fixed target can be recommended ${ }^{143}$. Some findings suggest individualizing blood pressure management by adjusting the MAP targets based on the typical blood pressure of the patient ${ }^{146}$. Similarly, patients with increased venous pressure (for example, owing to right-sided heart failure) may not achieve adequate perfusion pressure of the kidney at a
MAP of $65 \mathrm{mmHg}$. In addition, intra-abdominal hypertension is especially problematic for kidney perfusion because it can affect both arterial flow and venous pressure $^{147}$. Thus, clinicians must individualize the care of patients, sometimes trying a higher MAP in select cases. Optimizing volume status while simultaneously treating vasomotor paralysis with vasopressors is critical (FIG. 6). Studies using functional haemodynamic monitoring to guide haemodynamic management have shown promise in both cardiac surgery and sepsis ${ }^{148}$. Noradrenaline is the first-line choice as a vasopressor for vasodilatory shock $^{149}$. Other agents are generally reserved for refractory shock or for specific conditions and none of the agents is universally more 'kidney-friendly'. Angiotensin II may have advantages in some individuals who have an angiotensin II deficiency ${ }^{150}$, and corticosteroids are generally recommended as an adjunct therapy in patients with severe septic shock.

\section{Nephrotoxic drugs and agents}

Risk for AKI increases with the number of nephrotoxic drugs used and all potentially nephrotoxic agents that can be stopped should be discontinued ${ }^{5,50}$. Indispensable agents should only be used as long as needed and only at required doses. Careful monitoring of drug concentrations is also mandatory if possible (for example, for vancomycin). Arterial radiocontrast agents should be limited to situations where the therapeutic benefit outweighs the risk and should be used at the lowest volume possible, for example, omitting the ventriculography 
part of cardiac catheterization unless absolutely necessary. Finally, fluids containing non-physiologic ratios of sodium and chloride may worsen $\mathrm{AKI}^{151,152}$. Balanced electrolyte solutions, such as lactated Ringer's solution, are preferable in most patients.

\section{Stage-based management of AKI}

The 2012 KDIGO AKI guideline emphasizes the importance of AKI staging as a guide to management (FIG. 7$)^{1}$. Prognosis is strongly correlated with peak AKI stage and the duration of AKI (transient versus persistent) such that the urgency and invasiveness of diagnostic and therapeutic actions increases with AKI stage ${ }^{96}$. However, AKI stages must be interpreted in the context of baseline kidney function. For patients with previous normal kidney function and stage $1 \mathrm{AKI}$, management mainly involves rapid identification of the likely cause of AKI and avoidance of secondary insults. Depending on baseline GFR, adjusting drug dosage usually becomes clinically important at stage 2 AKI. Retained solutes are rarely of concern at stage 1 and stage 2 AKI unless the patient has clinically relevant underlying CKD. However, sodium excretion and fluid regulation may already be impaired and careful attention to volume input and output is recommended ${ }^{39}$. At stage 3 AKI, disruption of acid-base balance and electrolyte levels and accumulation of uraemic toxins may cause symptoms ${ }^{153}$. For example, patients may develop tachypnoea not only from fluid overload but also from metabolic acidosis. Acidosis will also shift potassium out of cells, further aggravating hyperkalaemia. Even relatively moderate uraemia may worsen platelet function and increase the risk of bleeding. Appropriate medical management of these conditions is effective in most patients $^{1}$ (Supplementary Fig. 1). In particular, judicious use of loop diuretics can be quite effective in augmenting sodium, potassium and fluid excretion ${ }^{154}$. However,

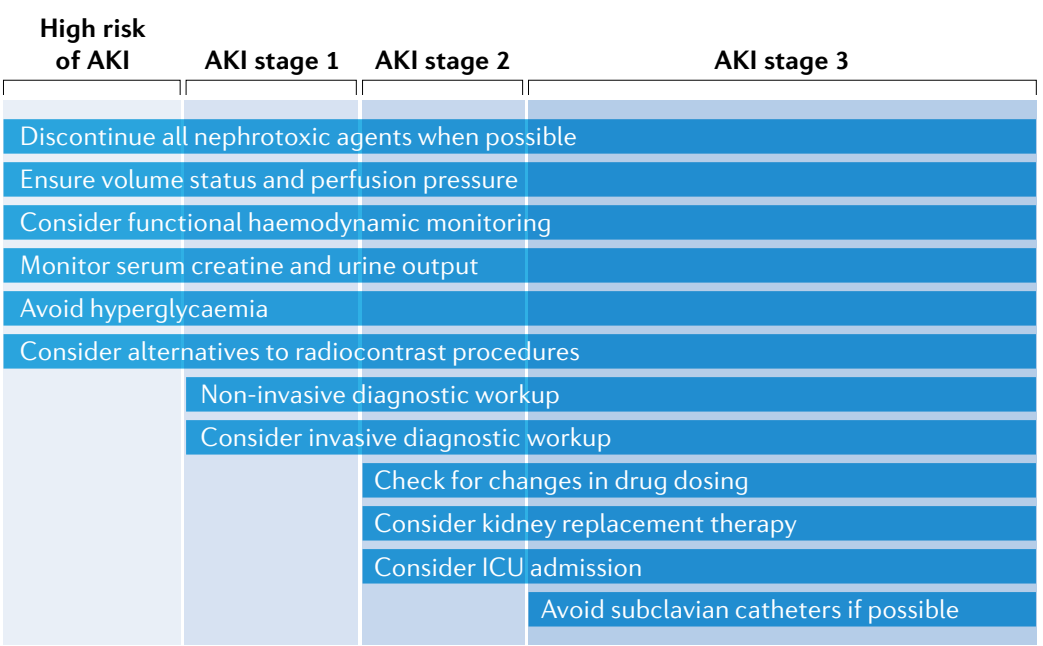

Fig. 7 | Management of AKI. The 2012 acute kidney injury (AKI) guidelines of Kidney Disease Improving Global Outcomes (KDIGO) group lists a series of actions in patients with $\mathrm{AKI}$ depending on $\mathrm{AKI}$ stage $\mathrm{e}^{1}$. Volume control is essential for patients at risk of AKI and all stages of AKI. A diagnostic work-up is warranted and dose adjustments of medications to the changing kidney excretory function are required. Decisions on if and when to start kidney replacement therapy and adequate monitoring requires expertise in critical care medicine and/or nephrology. ICU, intensive care unit. Adapted with permission from REF. ${ }^{1}$, Elsevier. when medical management is ineffective or if disruptions are life-threatening, KRT will be required.

At all stages of AKI, discontinuation of all potentially nephrotoxic medication as soon as possible is recommended, as all medications cause or contribute to AKI in most cases and are likely to be the most modifiable risk factors for $\mathrm{AKI}^{50}$. Importantly, not all drugs that affect kidney function are nephrotoxic but any drug that reduces renal function can complicate AKI and lead to adverse effects ${ }^{140}$. Thus, limiting exposure to such drugs as much as possible is imperative. Volume management and haemodynamic monitoring are also required at all stages of AKI. Avoidance of hyperglycaemia is important because the filtered glucose increases tubular reabsorption workload and oxidative stress, a process that sensitizes the kidney tubule to injury ${ }^{155}$. However, intensive insulin therapy often has adverse effects and management guidelines recommend maintaining blood glucose concentration at $110-149 \mathrm{mg} / \mathrm{dl}(6.1-8.3 \mathrm{mmol} / \mathrm{l})^{1}$. Finally, when in stage 3 AKI KRT becomes necessary, guidelines recommend avoiding the subclavian vein for KRT access, because this may lead to central vein stenosis and jeopardize subsequent permanent access ${ }^{156,157}$.

We note that evidence is accumulating that AKI leads to an increased risk of infection ${ }^{158,159}$. In experimental models, neutrophil function becomes impaired as early as stage $1 \mathrm{AKI}^{160}$, and uraemic toxins such as resistin may contribute to immune dysfunction ${ }^{161}$. Thus, patients with AKI should be monitored closely for sepsis.

An important and unanswered question is whether different forms of AKI can be treated with targeted approaches based on their underlying cause. This strategy is possible for obstructive uropathy or atypical haemolytic uraemic syndrome, but other forms of AKI often have an undefined cause. Achieving a molecular signature with specific biomarkers for different forms of AKI is a task for future research ${ }^{5}$. According to current knowledge, risk factors and risk modifiers - such as drugs, contrast media, low cardiac output conditions and congestion - should be reduced or eliminated. Even if the cause of the AKI episode can be recognized, this awareness might come too late to prevent initiation of the final common pathway of tubular toxicity, ischaemia and inflammation. Hence, it is essential to use the best available and new biomarkers to recognize initial AKI phases (stage 1S; TABLE 2) and to apply protective measures and risk mitigation to avoid worsening of the condition. Finally, even when AKI has fully developed (stage 2 and stage $3 \mathrm{AKI}$ ), identifying patients who might progress to AKD or even CKD is important ${ }^{140}$. In these patients, specific biomarkers may help with planning the allocation of resources and in identifying patients in whom antifibrotic agents can be used and blockade of endothelial-mesenchymal transition processes in the kidney tissue can be attempted ${ }^{5}$.

\section{Management across the trajectory of AKI}

In most patients with AKI who receive medical attention and in whom the injury is either self-limited (for example, surgery) or the underlying cause has been corrected (for example, nephrotoxic drug discontinued or infection treated), kidney function begins to improve within $24-48$ hours $^{162}$. However, in $25-35 \%$ of patients, 
Peritoneal dialysis

A treatment for kidney failure that uses the inside lining of the abdomen as a filtration membrane via intermittent filling and emptying of the peritoneal cavity with a dialysate solution to extract salt, uraemic toxins and other solutes from the blood.
AKI persists for $\geq 72$ hours $^{163}$. These patients have considerably worse outcomes. Thus, persistent AKI should prompt clinicians to revisit their working diagnosis as to the cause of AKI (BOX 1) and re-evaluate the general management principles ${ }^{140}$. For example, a patient who develops AKI following cardiac surgery should have volume status, haemodynamics, and medication list carefully reviewed, and any problems corrected. For most of these patients, this approach will be effective. However, if AKI persists, the clinician should check these points again to ensure that nothing was missed. For clinicians who have little experience in the care of patients with AKI, this might also involve consultation with a nephrologist ${ }^{140}$.

Recurrent or relapsing AKI is common, especially in patients in the $\mathrm{ICU}^{67}$. Whether recurrence is caused by distinct insults or relapsing kidney dysfunction owing to evolution of the injury from a single insult varies. In general, it is best to assume that recurrence is potentially due to a new cause that needs to be identified. Management of AKD without AKI is similar to that of AKD with AKI. However, these patients will often be identified in the community and a causative event is often not obvious. Risk factors and causes of AKD without AKI are poorly understood. Unless AKD resolves rapidly, management will also begin to include recommendations for CKD management ${ }^{140}$.

Recovery following AKI is best assessed after hospital discharge but follow-up monitoring of these patients has been historically poor ${ }^{164}$. Patients may be discharged with unstable kidney function and are, therefore, at an increased risk of drug-associated adverse events, given that most receive renally excreted drugs. Both treatment failures from underdosing in patients whose kidney function improves and toxic effects from overdosing in those whose kidney function worsens are common causes for hospital readmission. Thus, patients should be seen promptly by a nephrologist following hospital discharge to assess kidney function ${ }^{140}$. Finally, even patients who seem to have completely recovered following AKI or AKD may be at an increased risk of subsequent kidney injury for an unknown period. A prudent course is to be cautious with reinstitution of potentially nephrotoxic drugs and to monitor patients closely for 3-6 months after AKI or AKD recovery ${ }^{140}$.

\section{Kidney replacement therapy}

KRT and support have considerably evolved over the years, making application of extracorporeal therapies safer and easier. Controversies still exist on timing of initiation and selection of patients, often owing to heterogeneity of the studied populations ${ }^{140,165}$. The ADQI group has recommended an approach that matches the demand of blood purification with the kidney's capacity on a day-to-day basis; however, how to assess demand and capacity are not standardized ${ }^{5,140}$. Furthermore, interdisciplinary evaluation may further refine the criteria for initiation. In a patient-centred evaluation of the need to initiate KRT based on principles of precision medicine, life-threatening conditions are not the only indications and prevention of clinical complications should also be considered.
Peritoneal dialysis has been used for many years and is still used in regions where access to more sophisticated techniques is limited or these are not available ${ }^{166-169}$. In all other circumstances, extracorporeal techniques are preferred. In the absence of evidence supporting specific techniques, modality selection should be based on a pathophysiological rationale ${ }^{170}$. In patients whose condition is unstable and in those who are critically ill, continuous KRT is often preferred ${ }^{170}$. Once the patient is discharged from the ICU, intermittent techniques, such as sustained low efficiency dialysis or daily intermittent haemodialysis, can be safely used. Continuous veno-venous haemofiltration, continuous veno-venous haemodialysis or continuous veno-venous haemodiafiltration are used according to centre experience and personnel training rather than evidence-based difference between the techniques ${ }^{170}$. Beyond KRT, different membranes and additional devices, such as adsorbers, are available to expand blood purification to other circulating mediators of critical illness, although the scientific evidence for reducing mortality or other meaningful clinical outcomes are scarce. For example, high cut-off membranes enable removal of large molecules, such as free antibody light chains or myoglobin ${ }^{171}$. In patients with sepsis-associated AKI, a conceptual model has been proposed of sequential extracorporeal therapy with early removal of endotoxin by polymyxin-B haemoperfusion followed by removal of cytokines and pro-inflammatory and anti-inflammatory mediators by sorbent devices ${ }^{172}$. Subsequently, different configurations of the extracorporeal circuit may enable support of not only the kidney but also the heart, the lungs and the liver ${ }^{172}$. The best time point to start KRT in patients who are critically ill remains controversial because, in part, relevant trials have had conflicting results regarding kidney outcomes ${ }^{173-175}$. Indeed, best practice in KRT may differ in many ways, especially in specific populations.

Children and especially neonates with a body weight $<4 \mathrm{~kg}$ have high mortality in the case of AKI and may require dedicated KRT technology. A specific device called CARPEDIEM (Cardio Renal Paediatric Dialysis Emergency Machine) has been approved for continuous KRT in paediatric patients weighing $2.5-10 \mathrm{~kg}$ who are critically ill ${ }^{176}$. Use of this platform has simplified application of KRT in neonates, which may contribute to a $50 \%$ survival at ICU discharge in settings where the system is regularly applied ${ }^{177}$.

\section{Quality of life}

Data on the quality of life of patients with AKI during the ICU phase of the disease, at which point many patients receive narcotics during ventilation support, are lacking. AKI-specific aspects would also be difficult to assess given the frequent complexity of medical conditions. Available studies focus on the long-term effects of AKI on the health-related quality of life (HRQL) and functional status of survivors of critical illness. The 36-Item Short Form Health Survey (SF-36) and the EuroQol EQ-5D are the most frequently used HRQL instruments in this context. Most studies consistently show that survivors of AKI have substantially reduced 
HRQL compared with survivors of a critical illness without AKI or with the general population ${ }^{178,179}$; this result has also been found in children ${ }^{180}$. HRQL was lower in patients who required KRT for severe AKI than in those who did not require KRT and was largely driven by physical impairments ${ }^{181}$, for example, limited energy, diminished mobility, difficulty with ambulation or otherwise decreased physical fitness ${ }^{178}$. $20-40 \%$ of AKI survivors had a new disability in at least one of the activities of daily living and only $28-69 \%$ of AKI survivors who were employed before their critical illness were able to return to work ${ }^{178,182,183}$.

Long-term HRQL also relates to whether patients experience kidney function recovery or remain dialysis dependent. Quality of life is impaired in both groups, but one study found it to be substantially lower in those that remained on dialysis ${ }^{184}$. However, in many patients with AKI, HRQL had already been impaired at baseline and the difference in HRQL before and after AKI may be small ${ }^{178}$, which would be consistent with the observation that elderly and frail individuals are particularly susceptible to AKI ${ }^{185}$. The ethics of offering KRT to these patients in such a setting can be complex, involving predictions of benefits and harms, and projections of retrospective judgements. Interestingly, most AKI survivors (71.4-98.5\%) were satisfied with the care they had received and would agree to undergo the same treatment again, including $\mathrm{KRT}^{178}$. Indeed, at 1 year after AKI requiring dialysis, $81.8 \%$ of survivors would accept readmission to an ICU if necessary ${ }^{186}$, but this number declined to $71.4 \%$ after 4 years.

\section{Outlook}

Improvements in AKI diagnosis and treatment remain unmet medical needs. From a diagnostic perspective, implementing a kidney injury marker into clinical practice, compared with the current kidney function-based approach (which actually indicates acute kidney failure) remains a priority ${ }^{5}$. Measurement of serum creatinine levels does not enable the early diagnosis of AKI that is necessary to improve patient outcomes. In addition, creatinine assessment does not clarify to what extent subclinical AKI episodes contribute to the shortening of kidney lifespan and to CKD, and to the increased prevalence of hypertension, cardiovascular and cancer risk. Recommendations from the 23 rd consensus conference of the ADQI suggest that combining AKI definitions based on serum creatinine and urinary output with kidney injury biomarkers would improve the precision of AKI course prognostication ${ }^{5}$. To what extent novel biomarkers can help to improve short-term and long-term outcomes remains to be demonstrated in prospective trials.

New tools for kidney imaging can assist in defining AKI or specific causes of AKI ${ }^{187,188}$. In addition, measurement of GFR with suitable tracers or even online recording of GFR may be able to capture the dynamic changes of kidney function better in selected patients and these methods should be investigated ${ }^{189}$.

From a therapeutic perspective, drugs that can reduce tubular necrosis and save nephrons would be optimal to reduce the risk of death in the acute phase of AKI and to avoid development of CKD. Use of some experimental compounds improved short-term outcomes of AKI. For example, recombinant alkaline phosphatase improved creatinine clearance on day 28 in a small trial in patients with severe sepsis or septic shock ${ }^{190}$. However, these results did not repeat in a larger trial in 301 patients with sepsis-related AKI ${ }^{191}$. Development of more potent agents requires the filling of crucial gaps in the understanding of kidney pathophysiology after AKI. When and how tubular cell necrosis can be blocked without promoting the persistence of cells with substantial DNA damage eventually promoting malignancies needs to be addressed. Another important point that needs to be elucidated is why some patients who are in specific risk groups are predominantly affected by AKI, with particular focus on the identification of patients with subclinical AKI.

National and international research networks, consortia, and consensus panels can advance consensus on innovations at the international level. Examples of these efforts include the AKI-Epidemiologic Prospective Investigation (AKI-EPI) study ${ }^{192}$, the Southeast AsiaAKI (SEA-AKI) study ${ }^{193}$, the Assessment of Worldwide Acute Kidney Injury, Renal Angina, and Epidemiology (AWARE) $)^{103}$ and Assessment of Worldwide Acute Kidney Injury Epidemiology in Neonates (AWAKEN) studies $^{194}$, and the Acute Kidney Injury Clinical Study Group at KidneyResearchUK.

Primary prevention is also an essential focus of many initiatives. The National Institute for Health and Care Excellence (NICE) original guideline on AKI was published in 2013, following the observation that about 100,000 cases of AKI could have been averted across England with simple checks, such as ensuring patients are hydrated and that their medicines are reviewed, and that AKI costs were more than the National Health Service spends on breast, lung and skin cancer combined ${ }^{195}$. In 2013, the International Society of Nephrology launched the 0by 25 AKI Initiative to prevent all avoidable deaths from AKI across the world by $2025^{23,134}$. This initiative predominantly focuses on prevention of AKI in LMIC and low-resource settings, where KRT is not available or hardly accessible, with global outreach. Increasing the availability and accessibility for KRT is a priority in LMIC. Distinctive features of AKI in Western HIC are the ageing population, the association with multiple organ failure, the advanced technology available for patient care, the emerging area of onco-nephrology and the financial resources that enable care for almost all patients with $\mathrm{AKI}^{196}$. The European Renal Association European Dialysis and Transplant Association has created a network of nephrologists to organize, coordinate and improve the practices, research and education in the field of AKI in Europe ${ }^{197}$. Finally, new technologies for KRT and drug therapeutic approaches remain heterogeneous across regions and even between treatment centres. Lack of agreement and uniformity delays progress and a shared approach is required to improve understanding, diagnosis and treatment of AKI across the globe.

Published online: 15 July 2021 
1. KDIGO AKI Work Group. KDIGO clinical practice guideline for acute kidney injury. Kidney Int Suppl. 2 $1-138$ (2012)

2. Bhatraju, P. K. et al. Association between early recovery of kidney function after acute kidney injury and long-term clinical outcomes. JAMA Netw. Open 3 e202682 (2020).

3. Chu, R. et al. Assessment of KDIGO definitions in patients with histopathologic evidence of acute renal disease. Clin. J. Am. Soc. Nephrol. 9, 1175-1182 (2014).

4. Kane-Gill, S. L., Meersch, M. \& Bell, M. Biomarkerguided management of acute kidney injury. Curr. Opin. Crit. Care 26, 556-562 (2020)

5. Ostermann, M. et al. Recommendations on acute kidney injury biomarkers from the acute disease quality initiative consensus conference: a consensus statement. JAMA Netw. Open 3, e2019209 (2020).

6. Bhagwanani, A., Carpenter, R. \& Yusuf, A. Improving the management of acute kidney injury in a district general hospital: introduction of the DONUT bundle. BMJ Qual. Improv. Rep 2, u202650.w1235 (2014).

7. Biswas, A. et al. Identification of patients expected to benefit from electronic alerts for acute kidney injury. Clin. J. Am. Soc. Nephrol. 13, 842-849 (2018).

8. Joslin, J. et al. Recognition and management of acute kidney injury in hospitalised patients can be partially improved with the use of a care bundle. Clin. Med. 15 , 431-436 (2015)

9. Lachance, P. et al. Association between e-alert implementation for detection of acute kidney injury and outcomes: a systematic review. Nephrol. Dial. Transplant. 32, 265-272 (2017).

10. Wołyniec, W. et al. Glomerular filtration rate is unchanged by ultramarathon. J. Strength Cond. Res. 32, 3207-3215 (2018)

11. MacDonald, A. J., Nadim, M. K., Durand, F. \& Karvellas, C. J. Acute kidney injury in cirrhosis: implications for liver transplantation. Curr. Opin. Crit. Care 25, 171-178 (2019)

12. Fenoglio, R., Sciascia, S., Baldovino, S. \& Roccatello, D. Acute kidney injury associated with glomerular diseases. Curr. Opin. Crit. Care 25, 573-579 (2019).

13. Mannon, R. B. Delayed graft function: the AKI of kidney transplantation. Nephron 140, 94-98 (2018).

14. Selewski, D. T. et al. Neonatal acute kidney injury. Pediatrics 136, e463-e473 (2015)

15. Lewington, A. J., Cerdá, J. \& Mehta, R. L. Raising awareness of acute kidney injury: a global perspective of a silent killer. Kidney Int. 84, 457-467 (2013).

16. Cerdá, J., Bagga, A., Kher, V. ¿ Chakravarthi, R. M. The contrasting characteristics of acute kidney injury in developed and developing countries. Nat. Clin. Pract. Nephrol. 4, 138-153 (2008)

17. Luyckx, V. A., Tonelli, M. \& Stanifer, J. W. The global burden of kidney disease and the sustainable development goals. Bull. World Health Organ. 96 414-422 (2018)

18. Jha, V. \& Parameswaran, S. Community-acquired acute kidney injury in tropical countries. Nat. Rev. Nephrol. 9, 278-290 (2013).

19. Olowu, W. A. et al. Outcomes of acute kidney injury in children and adults in sub-Saharan Africa: a systematic review. Lancet Glob. Health $\mathbf{4}$ e242-e250 (2016)

20. Susantitaphong, P. et al. World incidence of AKI: a meta-analysis. Clin. J. Am. Soc. Nephrol. 8 1482-1493 (2013).

21. Martin-Cleary, C., Molinero-Casares, L. M., Ortiz, A. \& Arce-Obieta, J. M. Development and internal validation of a prediction model for hospital-acquired acute kidney injury. Clin. Kidney J. 14, 309-316 (2021).

22. Hoste, E. A. J. et al. Global epidemiology and outcomes of acute kidney injury. Nat. Rev. Nephrol. 14, 607-625 (2018)

23. Mehta, R. L. et al. International Society of Nephrology's 0by25 initiative for acute kidney injury (zero preventable deaths by 2025): a human rights case for nephrology. Lancet 385, 2616-2643 (2015)

24. Mehta, R. L. et al. Recognition and management of acute kidney injury in the International Society of Nephrology Oby25 Global Snapshot: a multinational cross-sectional study. Lancet 387, 2017-2025 (2016).

25. Bairey Merz, C. N. et al. Sex and the kidneys: current understanding and research opportunities. Nat. Rev. Nephrol. 15, 776-783 (2019).

26. Chew, S. T., Mar, W. M. \& Ti, L. K. Association of ethnicity and acute kidney injury after cardiac surgery in a South East Asian population. Br. J. Anaesth. 110, 397-401 (2013)
27. Beers, K. et al. Racial and ethnic disparities in pregnancy-related acute kidney injury. Kidney 3601 , 169-178 (2020)

28. Grams, M. E. et al. Explaining the racial difference in AKI incidence. J. Am. Soc. Nephrol. 25, 1834-1841 (2014).

29. Cerdā, J. et al. Epidemiology of acute kidney injury. Clin. J. Am. Soc. Nephrol. 3, 881-886 (2008).

30. Lameire, N., Van Biesen, W. \& Vanholder, R. The changing epidemiology of acute renal failure. Nat. Clin. Pract. Nephrol. 2, 364-377 (2006).

31. Lameire, N. H. et al. Acute kidney injury: an increasing global concern. Lancet 382, 170-179 (2013).

32. Kaul, A., Bhadauria, D., Prasad, N., Gupta, A. \& Sharma, R. K. Recurrent acute kidney injury in tropics - epidemiology and outcomes. J. Assoc. Physicians India 66, 18-21 (2018).

33. Uchino, $\mathrm{S}$. et al. Acute renal failure in critically ill patients: a multinational, multicenter study. JAMA 294, 813-818 (2005).

34. Denic, A. et al. The substantial loss of nephrons in healthy human kidneys with aging. J. Am. Soc. Nephrol. 28, 313-320 (2017).

35. Denic, A. et al. Single-nephron glomerular filtration rate in healthy adults. N. Engl. J. Med. 376 , 2349-2357 (2017).

36. Levey, A. S. et al. Nomenclature for kidney function and disease: report of a Kidney Disease: Improving Global Outcomes (KDIGO) consensus conference. Kidney Int. 97, 1117-1129 (2020)

37. Saran, R. et al. US Renal Data System 2019 annual data report: epidemiology of kidney disease in the United States. Am. J. Kidney Dis. 75, A6-A7 (2020).

38. Romagnani, P. et al. Chronic kidney disease. Nat. Rev. Dis. Prim. 3, 17088 (2017).

39. Prowle, J. R., Kirwan, C. J. \& Bellomo, R. Fluid management for the prevention and attenuation of acute kidney injury. Nat. Rev. Nephrol. 10, 37-47 (2014).

Overview of fluid status assessment and analysis of cardiovascular and renal targets for the prevention and attenuation of $A K I$.

40. Weyker, P. D., Pérez, X. L. \& Liu, K. D. Management of acute kidney injury and acid-base balance in the septic patient. Clin. Chest Med. 37, 277-288 (2016)

41. Lee, S. A., Cozzi, M., Bush, E. L. \& Rabb, H. Distant organ dysfunction in acute kidney injury: a review. Am. J. Kidney Dis. 72, 846-856 (2018).

42. Meijers, B., Evenepoel, P. \& Anders, H. J. Intestinal microbiome and fitness in kidney disease. Nat. Rev Nephrol. 15, 531-545 (2019).

43. Li, X., Hassoun, H. T., Santora, R \& Rabb, H. Organ crosstalk: the role of the kidney. Curr. Opin. Crit. Care 15, 481-487 (2009)

44. Faubel, S. \& Edelstein, C. L. Mechanisms and mediators of lung injury after acute kidney injury. Nat. Rev. Nephrol. 12, 48-60 (2016).

45. Nakazawa, D et al. Histones and neutrophil extracellular traps enhance tubular necrosis and remote organ injury in Ischemic AKI. J. Am. Soc Nephrol. 28, 1753-1768 (2017).

46. Di Lullo, L., Reeves, P. B., Bellasi, A. \& Ronco, C. Cardiorenal syndrome in acute kidney injury. Semin. Nephrol. 39, 31-40 (2019).

47. Kovalcikova, A. et al. Oxidative stress in the brain caused by acute kidney injury. Metab. Brain Dis. 33 961-967 (2018).

48. Sharfuddin, A. A. \& Molitoris, B. A. Pathophysiology of ischemic acute kidney injury. Nat. Rev. Nephrol. 7 189-200 (2011)

49. Ehrmann, S. et al. Nephrotoxic drug burden among 1001 critically ill patients: impact on acute kidney injury. Ann. Intensive Care 9, 106 (2019).

50. Goldstein, S. L. et al. Electronic health record identification of nephrotoxin exposure and associated acute kidney injury. Pediatrics 132, e756-e767 (2013).

51. Costa e Silva, V. T., Marçal, L. J. \& Burdmann, E. A. Risk factors for vancomycin nephrotoxicity: still a matter of debate*. Crit. Care Med. 42, 2635-2636 (2014).

52. Joyce, E. L., Kane-Gill, S. L., Priyanka, P. Fuhrman, D. Y. \& Kellum, J. A. Piperacillin/tazobactam and antibiotic-associated acute kidney injury in critically III children. J. Am. Soc. Nephrol. 30 2243-2251 (2019)

53. $\mathrm{Fu}, \mathrm{E}$. L. et al. Association of acute increases in plasma creatinine after renin-angiotensin blockade with subsequent outcomes. Clin. J. Am. Soc. Nephrol. 14 1336-1345 (2019).

54. Weisberg, L. S., Allgren, R. L., Genter, F. C. \& Kurnik, B. R. Cause of acute tubular necrosis affects its prognosis. The Auriculin Anaritide Acute Renal Failure Study Group. Arch. Intern. Med. 157, 1833-1838 (1997).

55. Santos, W. J. et al. Patients with ischaemic, mixed and nephrotoxic acute tubular necrosis in the intensive care unit-a homogeneous population? Crit. Care 10 R68 (2006).

56. Mulay, S. R. \& Anders, H. J. Crystal nephropathies: mechanisms of crystal-induced kidney injury. Nat. Rev Nephrol. 13, 226-240 (2017).

57. Kers, J., Leemans, J. C. \& Linkermann, A. An overview of pathways of regulated necrosis in acute kidney injury. Semin. Nephrol. 36, 139-152 (2016).

58. Linkermann, A. et al. Synchronized renal tubular cell death involves ferroptosis. Proc. Natl Acad. Sci. USA 111, 16836-16841 (2014).

Study demonstrating the occurrence of regulated necrosis and synchronized death of upon AKI, with consequent triggering of a detrimental immune response.

59. Arai, S. et al. Apoptosis inhibitor of macrophage protein enhances intraluminal debris clearance and ameliorates acute kidney injury in mice. Nat. Med. 22 183-193 (2016)

60. Salei, N. et al. The kidney contains ontogenetically distinct dendritic cell and macrophage subtypes throughout development that differ in their inflammatory properties. J. Am. Soc. Nephrol. 31, 257-278 (2020)

61. Linkermann, A., Stockwell, B. R., Krautwald, S. \& Anders, H. J. Regulated cell death and inflammation: an auto-amplification loop causes organ failure. Nat. Rev. Immunol. 14, 759-767 (2014).

62. Mulay, S. R., Linkermann, A. \& Anders, H. J. Necroinflammation in kidney disease. J. Am. Soc. Nephrol. 27, 27-39 (2016).

63. Lazzeri, E. et al. Endocycle-related tubular cell hypertrophy and progenitor proliferation recover renal function after acute kidney injury. Nat. Commun. 9 1344 (2018).

Study describing the occurrence of cellular hypertrophy in the absence of tissue reconstitution and limited regeneration after AKI

64. Rinkevich, Y. et al. In vivo clonal analysis reveals lineage-restricted progenitor characteristics in mammalian kidney development, maintenance, and regeneration. Cell Rep. 7, 1270-1283 (2014).

65. Kang, H. M. et al. Sox9-positive progenitor cells play a key role in renal tubule epithelial regeneration in mice. Cell Rep. 14, 861-871 (2016)

66. Chawla, L. S. et al. Acute kidney disease and renal recovery: consensus report of the Acute Disease Quality Initiative (ADOI) 16 Workgroup. Nat. Rev. Nephrol. 13, 241-257 (2017).

67. Kellum, J. A., Sileanu, F. E., Bihorac, A., Hoste, E. A. \& Chawla, L. S. Recovery after acute kidney injury. Am. J. Respir. Crit. Care Med. 195, 784-791 (2017).

68. Lazzeri, E., Angelotti, M. L., Conte, C., Anders, H. J. $\&$ Romagnani, P. Surviving acute organ failure: cell polyploidization and progenitor proliferation Trends Mol. Med. 25, 366-381 (2019).

69. Patel, S. S., Palant, C. E., Mahajan, V. \& Chawla, L. S. Sequelae of AKI. Best. Pract. Res. Clin. Anaesthesiol. 31, 415-425 (2017)

70. Silver, S. A. et al. Causes of death after a hospitalization with AKI. J. Am. Soc. Nephrol. 29 1001-1010 (2018).

Study showing that cancer-related and cardiovascular deaths occurred at substantially higher rates than in the general population after AKI

71. Newsome, B. B. et al. Long-term risk of mortality and end-stage renal disease among the elderly after small increases in serum creatinine level during hospitalization for acute myocardial infarction. Arch. Intern. Med. 168, 609-616 (2008).

72. Parr, S. K. et al. Acute kidney injury is a risk factor for subsequent proteinuria. Kidney Int. 93, 460-469 (2018).

73. He, L. et al. AKI on CKD: heightened injury, suppressed repair, and the underlying mechanisms. Kidney Int. 92, 1071-1083 (2017).

74. Hsu, C. Y. et al. Elevated BP after AKI. J. Am. Soc Nephrol. 27, 914-923 (2016). Study showing high incidence of hypertension after AKI

75. Gammelager, H. et al. Three-year risk of cardiovascular disease among intensive care patients with acute kidney injury: a population-based cohort study. Crit. Care 18, 492 (2014)

76. Wu, V. C. et al. Long-term risk of coronary events after AKI. J. Am. Soc. Nephrol. 25, 595-605 (2014). 
77. Odutayo, A. et al. AKI and long-term risk for cardiovascular events and mortality. J. Am. Soc. Nephrol. 28, 377-387 (2017).

78. Xue, J. L. et al. Incidence and mortality of acute renal failure in Medicare beneficiaries, 1992 to 2001. J. Am. Soc. Nephrol. 17, 1135-1142 (2006).

79. Loef, B. G. et al. Immediate postoperative renal function deterioration in cardiac surgical patients predicts in-hospital mortality and long-term survival. J. Am. Soc. Nephrol. 16, 195-200 (2005)

80. Coca, S. G., Yusuf, B., Shlipak, M. G., Garg, A. X \& Parikh, C. R. Long-term risk of mortality and other adverse outcomes after acute kidney injury: a systematic review and meta-analysis. $\mathrm{Am}$. $\mathrm{J}$. Kidney Dis. 53, 961-973 (2009).

81. Peired, A. J. et al. Acute kidney injury promotes development of papillary renal cell adenoma and carcinoma from renal progenitor cells. Sci. Transl. Med. 12, eaaw6003 (2020)

82. Zhou, X. et al. Acute kidney injury instigates malignant renal cell carcinoma via CXCR2 in mice with inactivated Trp53 and Pten in proximal tubular kidney epithelial cells. Cancer Res. 81, 2690-2702 (2021).

83. Verine, J. et al. Human de novo papillary renal-cell carcinomas in a kidney graft: evidence of recipient origin with adenoma-carcinoma sequence. $\mathrm{Am}$. J. Transpl. 13, 984-992 (2013).

84. Chertow, G. M., Burdick, E., Honour, M., Bonventre, J. V. $\&$ Bates, D. W. Acute kidney injury, mortality, length of stay, and costs in hospitalized patients. J. Am. Soc Nephrol. 16, 3365-3370 (2005).

85. Lassnigg, A. et al. Minimal changes of serum creatinine predict prognosis in patients after cardiothoracic surgery: a prospective cohort study. J. Am. Soc. Nephrol. 15, 1597-1605 (2004).

86. Vanmassenhove, J. et al. Urinary output and fractional excretion of sodium and urea as indicators of transient versus intrinsic acute kidney injury during early sepsis. Crit. Care 17, R234 (2013).

87. Hoste, E. A. et al. RIFLE criteria for acute kidney injury are associated with hospital mortality in critically ill patients: a cohort analysis. Crit. Care 10, R73 (2006)

88. Hsu, C. Y. et al. Nonrecovery of kidney function and death after acute on chronic renal failure. Clin. J. Am. Soc. Nephrol. 4, 891-898 (2009).

89. Hsu, C. Y et al. The risk of acute renal failure in patients with chronic kidney disease. Kidney Int. $\mathbf{7 4}$, 101-107 (2008).

90. Ishani, A. et al. Acute kidney injury increases risk of ESRD among elderly. J. Am. Soc. Nephrol. 20 , 223-228 (2009)

91. Lafrance, J. P., Djurdjev, O. \& Levin, A. Incidence and outcomes of acute kidney injury in a referred chronic kidney disease cohort. Nephrol. Dial. Transpl. 25 2203-2209 (2010)

92. Pannu, N. et al. Modification of outcomes after acute kidney injury by the presence of CKD. Am. J. Kidney Dis. 58, 206-213 (2011).

93. Waikar, S. S. \& Bonventre, J. V. Creatinine kinetics and the definition of acute kidney injury. J. Am. Soc. Nephrol. 20, 672-679 (2009).

94. Broce, J. C., Price, L. L., Liangos, O., Uhlig, K. \& Jaber, B. L. Hospital-acquired acute kidney injury: an analysis of nadir-to-peak serum creatinine increments stratified by baseline estimated GFR. Clin. J. Am. Soc. Nephrol. 6, 1556-1565 (2011).

95. Chen, J. J., Chang, C. H., Huang, Y. T. \& Kuo, G. Furosemide stress test as a predictive marker of acute kidney injury progression or renal replacement therapy: a systemic review and meta-analysis. Crit. Care 24, 202 (2020).

96. Kellum, J. A. et al. Classifying AKI by urine output versus serum creatinine level. J. Am. Soc. Nephrol. 26 2231-2238 (2015)

97. Priyanka, P. et al. The impact of acute kidney injury by serum creatinine or urine output criteria on majo adverse kidney events in cardiac surgery patients. J. Thorac. Cardiovasc. Surg. https://doi.org/10.1016/j. jtcvs.2019.11.137 (2020)

98. Pottel, H., Mottaghy, F. M., Zaman, Z. \& Martens, F. On the relationship between glomerular filtration rate and serum creatinine in children. Pediatr. Nephrol. 25 927-934 (2010)

99. Schwartz, G. J. \& Work, D. F. Measurement and estimation of GFR in children and adolescents. Clin. J. Am. Soc. Nephrol. 4, 1832-1843 (2009).

100. Schwartz, G. J. et al. New equations to estimate GFR in children with CKD. J. Am. Soc. Nephrol. 20, 629-637 (2009)

101. Schwartz, G. J., Haycock, G. B., Edelmann, C. M. Jr. $\&$ Spitzer, A. A simple estimate of glomerular filtration rate in children derived from body length and plasma creatinine. Pediatrics 58, 259-263 (1976).

102. Plotz, F. B., Bouma, A. B., van Wijk, J. A., Kneyber, M. C. $\&$ Bokenkamp, A. Pediatric acute kidney injury in the ICU: an independent evaluation of pRIFLE criteria. Intensive Care Med. 34, 1713-1717 (2008)

103. Kaddourah, A., Basu, R. K., Bagshaw, S. M. Goldstein, S. L. \& Investigators, A. Epidemiology of acute kidney injury in critically III children and young adults. N. Engl. J. Med. 376, 11-20 (2017).

104. Endre, Z. H. et al. Improved performance of urinary biomarkers of acute kidney injury in the critically ill by stratification for injury duration and baseline renal function. Kidney Int. 79, 1119-1130 (2011).

105. McCullough, P. A. et al. Implementation of novel biomarkers in the diagnosis, prognosis, and management of acute kidney injury: executive summary from the tenth consensus conference of the Acute Dialysis Quality Initiative (ADOI). Contrib. Nephrol. 182, 5-12 (2013).

106. Murray, P. T. et al. Potential use of biomarkers in acute kidney injury: report and summary of recommendations from the 10th Acute Dialysis Quality Initiative consensus conference. Kidney Int. 85, 513-521 (2014)

107. Bagshaw, S. M., Zappitelli, M. \& Chawla, L. S. Nove biomarkers of AKI: the challenges of progress 'amid the noise and the haste'. Nephrol. Dial. Transpl. 28 235-238 (2013)

108. Lameire, N. H., Vanholder, R. C. \& Van Biesen, W. A How to use biomarkers efficiently in acute kidney injury. Kidney Int 79, 1047-1050 (2011).

109. Vanmassenhove, J., Vanholder, R., Nagler, E. \& Van Biesen, W. Urinary and serum biomarkers for the diagnosis of acute kidney injury: an in-depth review of the literature. Nephrol. Dial. Transpl. 28, 254-273 (2013).

110. Goldstein, S. L. \& Chawla, L. S. Renal angina. Clin. J. Am. Soc. Nephrol. 5, 943-949 (2010).

111. Cai, L., Rubin, J., Han, W., Venge, P. \& Xu, S. The origin of multiple molecular forms in urine of $\mathrm{HNL} /$ NGAL. Clin. J. Am. Soc. Nephrol. 5, 2229-2235 (2010).

112. Devarajan, P. Review: neutrophil gelatinase-associated lipocalin: a troponin-like biomarker for human acute kidney injury. Nephrology 15, 419-428 (2010).

113. Mishra, P. K. et al. Long-term quality of life postacute kidney injury in cardiac surgery patients. Ann. Card. Anaesth. 21, 41-45 (2018)

114. Makris, K. et al. Urinary neutrophil gelatinaseassociated lipocalin (NGAL) as an early marker of acute kidney injury in critically ill multiple trauma patients. Clin. Chem. Lab. Med. 47, 79-82 (2009).

115. Mcllroy, D. R., Wagener, G. \& Lee, H. T. Neutrophil gelatinase-associated lipocalin and acute kidney injury after cardiac surgery: the effect of baseline renal function on diagnostic performance. Clin. J. Am. Soc. Nephrol. 5, 211-219 (2010).

116. Perrotti, A. et al. Neutrophil gelatinase-associated lipocalin as early predictor of acute kidney injury after cardiac surgery in adults with chronic kidney failure. Ann. Thorac. Surg. 99, 864-869 (2015).

117. Doi, K. et al. Plasma neutrophil gelatinase-associated lipocalin in acute kidney injury superimposed on chronic kidney disease after cardiac surgery: a multicenter prospective study. Crit. Care 17, R270 (2013).

118. Kashani, K. et al. Discovery and validation of cell cycle arrest biomarkers in human acute kidney injury. Crit. Care 17, R25 (2013)

119. Bihorac, A. \& Kellum, J. A. Acute kidney injury in 2014: a step towards understanding mechanisms of renal repair. Nat. Rev. Nephrol. 11, 74-75 (2015).

120. Hoste, E. A. et al. Urinary cell cycle arrest biomarkers and chitinase 3 -like protein 1 (CHI3L1) to detect acute kidney injury in the critically ill: a post hoc laboratory analysis on the FINNAKI cohort. Crit. Care 24, 144 (2020).

121. Waskowski, J. et al. (TIMP2) x (IGFBP7) as early renal biomarker for the prediction of acute kidney injury in aortic surgery (TIGER). A single center observational study. PLOS ONE 16, e0244658 (2021).

122. Witzgall, R. Are renal proximal tubular epithelial cells constantly prepared for an emergency? Focus on "the proliferation capacity of the renal proximal tubule involves the bulk of differentiated epithelial cells". Am. J. Physiol. Cell Physiol. 294, C1-C3 (2008).

123. Meersch, M. et al. Urinary TIMP-2 and IGFBP7 as early biomarkers of acute kidney injury and renal recovery following cardiac surgery. PLOS ONE 9, e93460 (2014).

124. Aregger, F. et al. Identification of IGFBP-7 by urinary proteomics as a novel prognostic marker in early acute kidney injury. Kidney Int. 85, 909-919 (2014).

125. Koyner, J. L. et al. Tissue inhibitor metalloproteinase-2 (TIMP-2)IGF-binding protein-7 (IGFBP7) levels are associated with adverse long-term outcomes in patients with AKI. J. Am. Soc. Nephrol. 26 1747-1754 (2015).

126. Joannidis, M. et al. Use of cell cycle arrest biomarkers in conjunction with classical markers of acute kidney injury. Crit. Care Med. 47, e820-e826 (2019).

127. Xie, Y. et al. Tissue inhibitor metalloproteinase-2 (TIMP-2) • IGF-binding protein-7 (IGFBP7) levels are associated with adverse outcomes in patients in the intensive care unit with acute kidney injury. Kidney Int 95, 1486-1493 (2019).

128. Ronco, C., Kellum, J. A. \& Haase, M. Subclinical AK is still AKI. Crit. Care 16, 313 (2012).

129. Haase, M. et al. The outcome of neutrophil gelatinaseassociated lipocalin-positive subclinical acute kidney injury: a multicenter pooled analysis of prospective studies. J. Am. Coll. Cardiol. 57, 1752-1761 (2011).

130. Endre, Z. H., Pickering, J. W. \& Walker, R. J. Clearance and beyond: the complementary roles of GFR measurement and injury biomarkers in acute kidney injury (AKI). Am. J. Physiol. Ren. Physiol. 301, F697-F707 (2011)

131. Rabito, C. A., Panico, F., Rubin, R., Tolkoff-Rubin, N. \& Teplick, R. Noninvasive, real-time monitoring of renal function during critical care. J. Am. Soc. Nephrol. 4, 1421-1428 (1994).

132. Herrera-Gutierrez, M. E. et al. Replacement of 24-h creatinine clearance by $2-h$ creatinine clearance in intensive care unit patients: a single-center study. Intensive Care Med. 33, 1900-1906 (2007).

133. Pickering, J. W., Frampton, C. M., Walker, R. J., Shaw, G. M. \& Endre, Z. H. Four hour creatinine clearance is better than plasma creatinine for monitoring renal function in critically ill patients. Crit. Care 16, R107 (2012).

134. Schieppati, A., Perico, N. \& Remuzzi, G. Eliminating treatable deaths due to acute kidney injury in resource-poor settings. Semin. Dial. 28, 193-197 (2015).

135. Meersch, M. et al. Prevention of cardiac surgeryassociated AKI by implementing the KDICO guidelines in high risk patients identified by biomarkers: the PrevAKI randomized controlled trial. Intensive Care Med. 43, 1551-1561 (2017).

136. Engelman, D. T. et al. Using urinary biomarkers to reduce acute kidney injury following cardiac surgery. J. Thorac. Cardiovasc. Surg. 160, 1235-1246.e2 (2020).

137. Göcze, I. et al. Biomarker-guided intervention to prevent acute kidney injury after major surgery: the prospective randomized BigpAK Study. Ann. Surg. 267, 1013-1020 (2018).

138. Küllmar, M. et al. A multinational observational study exploring adherence with the kidney disease: improving global outcomes recommendations for prevention of acute kidney injury after cardiac surgery. Anesth. Analg. 130, 910-916 (2020)

139. Al-Jaghbeer, M., Dealmeida, D., Bilderback, A. Ambrosino, R. \& Kellum, J. A. Clinical decision support for in-hospital AKI. J. Am. Soc. Nephrol. 29, 654-660 (2018).

140. Ostermann, M. et al. Controversies in acute kidney injury: conclusions from a Kidney Disease: Improving Global Outcomes (KDIGO) conference. Kidney Int. 98 294-309 (2020)

141. Prowle, J. R., Echeverri, J. E., Ligabo, E. V., Ronco, C. $\&$ Bellomo, R. Fluid balance and acute kidney injury. Nat. Rev. Nephrol. 6, 107-115 (2010).

142. Busse, L. W. \& Ostermann, M. Vasopressor therapy and blood pressure management in the setting of acute kidney injury. Semin. Nephrol. 39, 462-472 (2019).

143. Xu, J. Y. et al. A high mean arterial pressure target is associated with improved microcirculation in septic shock patients with previous hypertension: a prospective open label study. Crit. Care 19, 130 (2015).

144. Asfar, P. et al. High versus low blood-pressure target in patients with septic shock. N. Engl. J. Med. 370 , 1583-1593 (2014).

145. Lamontagne, F. et al. Pooled analysis of higher versus lower blood pressure targets for vasopressor therapy septic and vasodilatory shock. Intensive Care Med. 44, 12-21 (2018). 
146. Futier, E. et al. Effect of individualized vs standard blood pressure management strategies on postoperative organ dysfunction among high-risk patients undergoing major surgery: a randomized clinical trial. JAMA 318, 1346-1357 (2017).

147. Dalfino, L., Tullo, L., Donadio, I., Malcangi, V. \& Brienza, N. Intra-abdominal hypertension and acute renal failure in critically ill patients. Intensive Care Med. 34, 707-713 (2008)

148. Pinsky, M. R. Functional hemodynamic monitoring. Crit. Care Clin. 31, 89-111 (2015).

149. Scheeren, T. W. L. et al. Current use of vasopressors in septic shock. Ann. Intensive Care 9, 20 (2019).

150. Rodriguez, R., Cucci, M., Kane, S., Fernandez, E. $\S$ Benken, S. Novel vasopressors in the treatment of vasodilatory shock: a systematic review of angiotensin II, selepressin, and terlipressin. J. Intensive Care Med. 35, 327-337 (2020)

151. Semler, M. W. et al. Balanced crystalloids versus saline in critically ill adults. N. Engl. J. Med. 378, 829-839 (2018)

152. Self, W. H. et al. Balanced crystalloids versus saline in noncritically ill adults. N. Engl. J. Med. 378, 819-828 (2018).

153. Bellomo, R., Kellum, J. A. \& Ronco, C. Acute kidney injury. Lancet 380, 756-766 (2012).

154. Patschan, D., Patschan, S., Buschmann, I. \& Ritter, O. Loop diuretics in acute kidney injury prevention, therapy, and risk stratification. Kidney Blood Press Res. 44, 457-464 (2019).

155. Peng, J. et al. Hyperglycemia, p53, and mitochondrial pathway of apoptosis are involved in the susceptibility of diabetic models to ischemic acute kidney injury. Kidney Int. 87, 137-150 (2015)

156. Lok, C. E. et al. KDOOI clinical practice guideline for vascular access: 2019 update. Am. J. Kidney Dis. 75 S1-s164 (2020)

157. O'Grady, N. P. et al. Guidelines for the prevention of intravascular catheter-related infections. Clin. Infect. Dis. 52, e162-e193 (2011).

158. Mehta, R. L. et al. Sepsis as a cause and consequence of acute kidney injury: program to improve care in acute renal disease. Intensive Care Med. 37 241-248 (2011).

159. Formeck, C. L., Joyce, E. L., Fuhrman, D. Y. \& Kellum, J. A. Association of acute kidney injury with subsequent sepsis in critically III children. Pediatr. Crit. Care Med. 22, e58-e66 (2020)

160. Singbartl, K. et al. Differential effects of kidney-lung cross-talk during acute kidney injury and bacterial pneumonia. Kidney Int. 80, 633-644 (2011).

161. Singbartl, K., Miller, L., Ruiz-Velasco, V. \& Kellum, J. A Reversal of acute kidney injury-induced neutrophil dysfunction: a critical role for resistin. Crit. Care Med. 44, e492-e501 (2016)

162. Palant, C. E., Patel, S. S. \& Chawla, L. S. Acute kidney injury recovery. Contrib. Nephrol. 193, 35-44 (2018).

163. Hoste, E. et al. Identification and validation of biomarkers of persistent acute kidney injury: the RUBY study. Intensive Care Med. 46, 943-953 (2020).

164. Karsanji, D. J. et al. Disparity between nephrologists' opinions and contemporary practices for community follow-up after AKI hospitalization. Clin. J. Am. Soc. Nephrol. 12, 1753-1761 (2017).

165. Kashani, K. et al. Quality improvement goals for acute kidney injury. Clin. J. Am. Soc. Nephrol. 14, 941-953 (2019).

166. McCulloch, M. et al. Challenges of access to kidney care for children in low-resource settings. Nat. Rev. Nephrol. 17, 33-45 (2021).

167. Callegari, J. et al. Peritoneal dialysis as a mode of treatment for acute kidney injury in sub-Saharan Africa. Blood Purif. 36, 226-230 (2013).

168. Cullis, B. et al. ISPD guidelines for peritoneal dialysis in acute kidney injury: 2020 update (adults). Perit. Dial. 41, 15-31 (2021).

169. Ponce, D., Berbel, M. N., Abrão, J. M., Goes, C. R. \& Balbi, A. L. A randomized clinical trial of high volume peritoneal dialysis versus extended daily hemodialysis for acute kidney injury patients. Int. Urol. Nephrol. 45, 869-878 (2013).

170. Karkar, A. \& Ronco, C. Prescription of CRRT: pathway to optimize therapy. Ann. Intensive Care 10 $32(2020)$

171. Balgobin, S. et al. Continuous veno-venous high cut-off hemodialysis compared to continuous veno-venous hemodiafiltration in intensive care unit acute kidney injury patients. Blood Purif. 46, 248-256 (2018).

172. De Rosa, S., Villa, G. \& Ronco, C. The golden hour of polymyxin $\mathrm{B}$ hemoperfusion in endotoxic shock: the basis for sequential extracorporeal therapy in sepsis. Artif. Organs 44, 184-186 (2020)

173. Zarbock, A. et al. Effect of early vs delayed initiation of renal replacement therapy on mortality in critically III patients with acute kidney injury: the ELAIN randomized clinical trial. JAMA 315, 2190-2199 (2016).

174. Gaudry, S. et al. Timing of renal support and outcome of septic shock and acute respiratory distress syndrome. a post hoc analysis of the AKIKI randomized clinical trial. Am. J. Respir. Crit. Care Med. 198, 58-66 (2018)

175. Barbar, S. D. et al. Timing of renal-replacement therapy in patients with acute kidney injury and sepsis N. Engl. J. Med. 379, 1431-1442 (2018). Study showing that among critically ill patients with AKI an accelerated renal replacement strategy is not associated with a lower risk of death than a standard strategy

176. Ronco, C. et al. Continuous renal replacement therapy in neonates and small infants: development and first-in-human use of a miniaturised machine (CARPEDIEM). Lancet 383, 1807-1813 (2014). Study reporting a new miniaturised machine that can provide various dialytic treatments and support for multiple organ dysfunction in neonates and small infants.

177. Garzotto, F. et al. Continuous kidney replacement therapy in critically ill neonates and infants: a retrospective analysis of clinical results with a dedicated device. Pediatr. Nephrol. 35, 1699-1705 (2020).

178. Villeneuve, P. M., Clark, E. G., Sikora, L., Sood, M. M. $\&$ Bagshaw, S. M. Health-related quality-of-life among survivors of acute kidney injury in the intensive care unit: a systematic review. Intensive Care Med. $\mathbf{4 2}$ 137-146 (2016).

Study reporting that physical limitations and disabilities were more commonly exhibited by AKI patients

179. Stengel, B. et al. Risk profile, quality of life and care of patients with moderate and advanced CKD: The French CKD-REIN Cohort Study. Nephrol. Dial. Transpl. 34, 277-286 (2019).

180. Richardson, K. L., Watson, R. S. \& Hingorani, S. Quality of life following hospitalization-associated acute kidney injury in children. J. Nephrol. 31 249-256 (2018)

181. Korkeila, M., Ruokonen, E. \& Takala, J. Costs of care long-term prognosis and quality of life in patients requiring renal replacement therapy during intensive care. Intensive Care Med. 26, 1824-1831 (2000).

182. Morsch, C., Thomé, F. S., Balbinotto, A., Guimarães, J. F $\&$ Barros, E. G. Health-related quality of life and dialysis dependence in critically ill patient survivors of acute kidney injury. Ren. Fail. 33, 949-956 (2011).

183. Morgera, S., Kraft, A. K., Siebert, G., Luft, F. C. $\&$ Neumayer, H. H. Long-term outcomes in acute renal failure patients treated with continuous renal replacement therapies. Am. J. Kidney Dis. 40, 275-279 (2002)

184. Wang, A. Y. et al. Health-related quality of life in survivors of acute kidney injury: the prolonged outcomes study of the randomized evaluation of normal versus augmented level replacement therapy study outcomes. Nephrology 20, 492-498 (2015).

185. Akbar, S. \& Moss, A. H. The ethics of offering dialysis for AKI to the older patient: time to re-evaluate? Clin. J. Am. Soc. Nephrol. 9, 1652-1656 (2014).

186. Oeyen, S. et al. Long-term quality of life in critically ill patients with acute kidney injury treated with renal replacement therapy: a matched cohort study. Crit. Care 19, 289 (2015)

187. Charlton, J. R. et al. Magnetic resonance imaging accurately tracks kidney pathology and heterogeneity in the transition from acute kidney injury to chronic kidney disease Kidney Int 99, 173-185 (2021).

188. Huang, J., Li, J., Lyu, Y., Miao, Q. \& Pu, K. Molecular optical imaging probes for early diagnosis of druginduced acute kidney injury. Nat. Mater. 18, 1133-1143 (2019)

189. Schneider, A. G. \& Molitoris, B. A. Real-time glomerular filtration rate: improving sensitivity, accuracy and prognostic value in acute kidney injury. Curr. Opin. Crit. Care 26, 549-555 (2020).

190. Pickkers, P. et al. Alkaline phosphatase for treatment of sepsis-induced acute kidney injury: a prospective randomized double-blind placebo-controlled trial. Crit. Care 16, R14 (2012)

191. Pickkers, P. et al. Effect of human recombinant alkaline phosphatase on 7-day creatinine clearance in patients with sepsis-associated acute kidney injury: a randomized clinical trial. JAMA 320, 1998-2009 (2018).

192. Hoste, E. A. et al. Epidemiology of acute kidney injury in critically ill patients: the multinational AKI-EPI study. Intensive Care Med. 41, 1411-1423 (2015).

193. Srisawat, N. et al. The epidemiology and characteristics of acute kidney injury in the Southeast Asia intensive care unit: a prospective multicentre study. Nephrol. Dial. Transpl. 35, 1729-1738 (2020).

194. Jetton, J. G. et al. Incidence and outcomes of neonatal acute kidney injury (AWAKEN): a multicentre, multinational, observational cohort study. Lancet Child. Adolesc. Health 1, 184-194 (2017). Study reporting a high incidence of AKI in neonatal setting and its role as a common and independent risk factor for mortality and longer hospital stay.

195. National Clinical Guideline Centre. in Acute Kidney Injury: Prevention, Detection and Management Up to the Point of Renal Replacement Therapy 1-26 (National Clinical Guideline Centre, 2013).

196. Rondeau, E., Faguer, S. \& Robert, T. Advocacy for a European network of renal intensive care units. Nephrol. Dial. Transpl. 34, 1262-1264 (2019).

197. Massy, Z. A. et al. Nephrology and public policy committee propositions to stimulate research collaboration in adults and children in Europe. Nephrol. Dial. Transpl. 34, 1469-1480 (2019).

198. Kidney Disease: Improving Global Outcomes (KDIGO) CKD Work Group. KDIGO clinical practice guideline for the evaluation and management of chronic kidney disease. Kidney Int. Suppl. 3, 1-150 (2013).

199. Scheel, P. J., Liu, M. \& Rabb, H. Uremic lung: new insights into a forgotten condition. Kidney Int. 74 849-851 (2008)

200. Chan, L. et al. AKI in hospitalized patients with COVID-19. J. Am. Soc. Nephrol. 32, 151-160 (2020). Study reporting the high incidence of AKI in patients with Covid-19.

201. Hirsch, J. S. et al. Acute kidney injury in patients hospitalized with COVID-19. Kidney Int. 98, 209-218 (2020).

202. Chan, L. \& Coca, S. G. Acute kidney injury in the time of COVID-19. Kidney 360 1, 588-590 (2020).

203. Price-Haywood, E. G., Burton, J., Fort, D. \& Seoane, L. Hospitalization and mortality among black patients and white patients with Covid-19. N. Engl. J. Med. 382, 2534-2543 (2020)

204. Su, H. et al. Renal histopathological analysis of 26 postmortem findings of patients with COVID-19 in China. Kidney Int. 98, 219-227 (2020).

205. Nicolai, L. et al. Immunothrombotic dysregulation in covid- 19 pneumonia is associated with respiratory failure and coagulopathy. Circulation 142 , 1176-1189 (2020).

206. Santoriello, D. et al. Postmortem kidney pathology findings in patients with COVID-19. J. Am. Soc Nephrol. 31, 2158-2167 (2020).

207. Braun, F. et al. SARS-CoV-2 renal tropism associates with acute kidney injury. Lancet 396, 597-598 (2020).

208. Roufosse, C. et al. Electron microscopic investigations in COVID-19: not all crowns are coronas. Kidney Int 98, 505-506 (2020).

209. Nadim, M. K. et al. COVID-19-associated acute kidney injury: consensus report of the 25th Acute Disease Quality Initiative (ADQI) Workgroup. Nat. Rev. Nephrol. 16, 747-764 (2020).

210. Gupta, S et al. AKI treated with renal replacement therapy in critically ill patients with COVID-19. J. Am. Soc. Nephrol. 32, 161-176 (2020).

\section{Acknowledgements}

The European Research Council under the Consolidator Grant RENOIR supported P.R. (ERC-2014-CoG, grant number 648274). The Deutsche Forschungsgemeinschaft supported H.- J.A. (AN372/14-4, AN372/16-2, AN372/20-2, AN372/27-1 and AN372/30-1) and A.Z. (KFO342/1, ZA428/18-1 and ZA428/21-1). J.A.K. is supported by a grant (UH3DK114861) from the National Institute of Diabetes and Kidney Disease (NIDDK).

\section{Author contributions}

Sections of the Primer were written by the authors as follows: Introduction (H.-J.A., J.A.K.); Epidemiology (H.-J.A., J.A.K., G.A.); Mechanisms/pathophysiology (P.R., H.-J.A.); Diagnosis, screening and prevention (H.-J.A., A.Z.); Management (H.-J.A., J.A.K., C.R.); Quality of life (H.J.A.); Outlook (H.-J.A., P.R.); Overview of the Primer (H.J.A.). 


\section{Competing interests}

H.-J.A. received consultancy fees from Bayer, Boehringer, AstraZeneca, Janssen, Novartis, GlaxoSmithKline, Previpharma, Inositec and Secarna, unrelated to this publication. J.A.K. received consulting fees and/or grant support from Baxter, NxStage and Astute Medical/BioMerieux. J.A.K became a full-time employee of Spectral Medical after submission of this manuscript. C.R. has been consulting or part of advisory boards for Asahi Kasei Pharma, Astute, Baxter Biomerieux, B. Braun, Cytosorbents, Estor, Fresenius Medical Care, General Electric, Jafron, Medtronic and Toray. A.Z. has received consulting and/or lecture fees from Astute Medical/ BioMerieux, Fresenius, Baxter, AM Pharma, La Jolla Pharmaceuticals and Astellas. A.Z. has received grant sup port from Astute Medical/BioMerieux, unrelated to this publication. G.A. and P.R. declare no competing interests.

\section{Peer review information}

Nature Reviews Disease Primers thanks M. Ostermann R. Evans, E. Daher, who co-reviewed with G. C. Meneses, K. Doi, J. Prowle, S. Menez, D. Ponce, and the other, anonymous, reviewer(s) for their contribution to the peer review of this work.

\section{Publisher's note}

Springer Nature remains neutral with regard to jurisdictional claims in published maps and institutional affiliations.

Supplementary information

The online version contains supplementary material available at https://doi.org/10.1038/s41572-021-00284-z.

(c) Springer Nature Limited 2021 